\title{
A generalized integrability problem for $G$-structures
}

\author{
Andrea Santi ${ }^{1}$
}

Received: 26 January 2015 / Accepted: 9 July 2015 / Published online: 31 July 2015

(C) Fondazione Annali di Matematica Pura ed Applicata and Springer-Verlag Berlin Heidelberg 2015

\begin{abstract}
Given an $\widetilde{n}$-dimensional manifold $\widetilde{M}$ equipped with a $\widetilde{G}$-structure $\widetilde{\pi}: \widetilde{P} \rightarrow \widetilde{M}$, there is a naturally induced $G$-structure $\pi: P \rightarrow M$ on any submanifold $M \subset \widetilde{M}$ that satisfies appropriate regularity conditions. We study generalized integrability problems for a given $G$-structure $\pi: P \rightarrow M$, namely the questions of whether it is locally equivalent to induced $G$-structures on regular submanifolds of homogeneous $\widetilde{G}$-structures $\widetilde{\pi}: \widetilde{P} \rightarrow \widetilde{H} / \widetilde{K}$. If $\widetilde{\pi}: \widetilde{P} \rightarrow \widetilde{H} / \widetilde{K}$ is flat $k$-reductive, we introduce a sequence of generalized curvatures taking values in appropriate cohomology groups and prove that the vanishing of these curvatures is a necessary and sufficient condition for the solution of the corresponding generalized integrability problem.
\end{abstract}

Keywords G-structures · Generalized integrability problem ·

Generalized $(G, \widetilde{G})$-curvatures · Generalized Spencer cohomology groups

Mathematics Subject Classification 53C10 - 53B25 - 53A55 - 53A30

\section{Introduction}

Let $M$ be an $n$-dimensional manifold and $\pi: L(M) \rightarrow M$ the linear frame bundle of $M$, that is, the $\mathrm{GL}_{n}(\mathbb{R})$-bundle of all linear frames $u=\left(e_{i}\right)$ of the tangent spaces of $M$. We recall that, given a closed subgroup $G \subset \mathrm{GL}_{n}(\mathbb{R})$, a $G$-structure on $M$ is a $G$-reduction $P$ of $L(M)$, a bundle formed by a collection of linear frames with the property that any two of them $u=\left(e_{i}\right), u^{\prime}=\left(e_{i}^{\prime}\right)$ at a point $x \in M$ satisfy an equality of the form

$$
e_{j}^{\prime}=\sum_{i=1}^{n} A_{j}^{i} e_{i}, \quad \text { for some } A=\left(A_{j}^{i}\right) \in G
$$

Andrea Santi

asanti.math@gmail.com; andrea.santi@unipr.it

1 Dipartimento di Matematica e Informatica, Universitá di Parma, Parco Area delle Scienze 53/A, 43124 Parma, Italy 
(see, e.g., $[9,22])$. Any $G$-structure is naturally endowed with the so-called soldering form $\vartheta: T P \rightarrow \mathbb{R}^{n}$, defined by $\vartheta_{u}(v)=\left(v^{1}, \ldots, v^{n}\right)$, where the $v^{i}$ are the components of the vector $\pi_{*}(v) \in T_{\pi(u)} M$ w.r.t. the frame $u=\left(e_{i}\right)$.

One of the simplest examples of a $G$-structure is given by the flat $G$-structure $P^{o}$ on $\mathbb{R}^{n}$, the collection of all linear frames $\left(e_{i}\right)$ of the form

$$
e_{i}=\left.\sum_{j=1}^{n} A_{i}^{j} \frac{\partial}{\partial x^{j}}\right|_{x}, \quad\left(A_{i}^{j}\right) \in G, \quad\left(x^{i}\right)=\text { standard coord. of } \mathbb{R}^{n} .
$$

An arbitrary $G$-structure $\pi: P \rightarrow M$ is called (locally) integrable if it satisfies the following: Around any $x \in M$, there exists a local diffeomorphism $f: \mathcal{U} \rightarrow \mathbb{R}^{n}$ such that the bundle $f_{*}(P \mid \mathcal{U})$ of pushed-forward frames coincides with $\left.P^{o}\right|_{f(\mathcal{U})}$. Equivalently one may say that $P$ is locally integrable if $M$ can be covered by charts $\xi=\left(y^{i}\right)$ with the property that the coordinate frames $\left(\left.\frac{\partial}{\partial y^{i}}\right|_{x}\right)$ are in $P$ at all points. The problem of determining whether a given $G$-structure $\pi: P \rightarrow M$ is integrable or not is usually called the integrability problem. When $G \subset \mathrm{GL}_{n}(\mathbb{R})$ is of finite type, such problem is completely solvable. Indeed, it turns out that, in this case, $P$ is integrable if and only if all elements of a special collection of $G$-equivariant maps in the Spencer cohomology groups $H^{p, 2}(\mathfrak{g}), \mathfrak{g}=\operatorname{Li} e(G)$, are trivial (see [7]).

Another natural construction of $G$-structures is the following. Let $\tilde{\pi}: \widetilde{P} \rightarrow \widetilde{M}$ be a $\widetilde{G}$ structure on a manifold $\widetilde{M}$ of dimension $\widetilde{n}=n+m$ and $M \subset \widetilde{M}$ a submanifold of dimension $n$. We say that $M$ is $\widetilde{P}$-regular if the collection of frames $u \in \widetilde{P}$ that are adapted to $M$ (i.e., with $x=\tilde{\pi}(u) \in M$ and the first $n$ vectors tangent to $M$ ) constitutes a principal bundle $\pi_{\sharp}=\left.\tilde{\pi}\right|_{P_{\sharp}}: P_{\sharp} \rightarrow M$ with structure group

$$
G_{\sharp}=\left\{\widetilde{A}=\left(\begin{array}{ll}
A & B \\
0 & D
\end{array}\right) \in \widetilde{G} \mid A \text { size } n \times n\right\} .
$$

Note that the quotient $G_{\sharp} / N_{\sharp}$ of $G_{\sharp}$ by the closed and normal subgroup

$$
N_{\sharp}=\left\{\widetilde{A}=\left(\begin{array}{ll}
I & B \\
0 & D
\end{array}\right) \in \widetilde{G}\right\}
$$

is isomorphic to $G=\left\{A \in \mathrm{GL}_{n}(\mathbb{R}) \mid A=\right.$ upper left block of some $\left.\widetilde{A} \in G_{\sharp}\right\}$. If $M \subset \widetilde{M}$ is $\widetilde{P}$-regular, the quotient bundle $\pi: P=P_{\sharp} / N_{\sharp} \rightarrow M$ with structure group $G=G_{\sharp} / N_{\sharp}$ is naturally identifiable with the $G$-structure on $M$ given by the frames formed by the first $n$ vectors of the adapted frames. We call it $G$-structure induced by $\widetilde{P}$; its frames $u \in P$ are the induced frames. First examples of such induced structures are the orthonormal frame bundles of $n$-dimensional submanifolds of the flat Riemannian manifold $\mathbb{R}^{n+m}$.

Now, given a $G$-structure $\pi: P \rightarrow M$ and an homogeneous $\widetilde{G}$-structure $\widetilde{\pi}: \widetilde{P} \rightarrow \widetilde{M}$ on a manifold of dimension $\widetilde{n}=n+m$, one may ask whether around any $x \in M$ there is a local embedding $f: \mathcal{U} \subset M \rightarrow \widetilde{M}$ such that $f(\mathcal{U})$ is $\widetilde{P}$-regular, and the pushed-forward bundle $f_{*}(P \mid \mathcal{U})$ is the $G$-structure induced by $\widetilde{P}$. If this occurs, we say that $f$ is a $(P, \widetilde{P})$ regular embedding and that $P$ is locally immersible into $(\widetilde{M}, \widetilde{P})$ (shortly, immersible in $\widetilde{P}$ ). By analogy with the classical integrability problem, we call the question on the existence of such local immersions the generalized integrability problem.

A crucial tool to deal with a given generalized integrability problem is provided by the following theorem.

Let $\widetilde{M}=\widetilde{H} / \widetilde{K}$ be an $\widetilde{n}$-dimensional homogeneous manifold with effective action and $\widetilde{\pi}: \widetilde{P} \rightarrow \widetilde{M}$ one of its associated homogeneous $\widetilde{G}$-structures. Each of them is a subbundle 
$\widetilde{P} \subset L(\widetilde{M})$ given by all frames of an orbit in $L(\tilde{M})$ of the group of diffeomorphisms $\widetilde{H} \subset \operatorname{Diff}(\widetilde{M})$ (see Sect. 2.1 for details). Let also $\widetilde{\mathfrak{h}}=\operatorname{Lie}(\widetilde{H}), \widetilde{\mathfrak{k}}=\operatorname{Lie}(\widetilde{K})$ and denote by $\widetilde{p}: \widetilde{\mathfrak{h}} \rightarrow \widetilde{\mathfrak{h}} / \widetilde{\mathfrak{k}} \simeq T_{o} \widetilde{M}, o=e \widetilde{K}$, the canonical projection onto $\widetilde{\mathfrak{h}} / \widetilde{\mathfrak{k}}$. In the following, the space $T_{o} \widetilde{M}$ is identified with $\mathbb{R}^{\widetilde{n}}$ by means of a linear frame $\left.u_{o} \in \widetilde{P}\right|_{o}$.

Theorem 1.1 Let $\pi: P \rightarrow M$ be a $G$-structure on an $n$-dimensional manifold $M$ with soldering form $\vartheta=\left(\vartheta^{1}, \ldots, \vartheta^{n}\right)$. Then, $P$ is locally immersible in $(\widetilde{M}=\widetilde{H} / \widetilde{K}, \widetilde{P})$ if and only if

(a) $G \simeq G_{\sharp} / N_{\sharp}$, where $G_{\sharp}, N_{\sharp} \subset \widetilde{G}$ are as in (1.1) and (1.2) respectively;

(b) for any local section $s: \mathcal{U} \subset M \rightarrow P$, there exists an $\widetilde{\mathfrak{h}}$-valued local 1-form $\omega$ on $M$ which satisfies

$$
\tilde{p} \circ \omega=\left(s^{*} \vartheta^{1}, \ldots, s^{*} \vartheta^{n}, 0, \ldots, 0\right)
$$

and

$$
d \omega+\frac{1}{2}[\omega, \omega]=0 .
$$

Furthermore, if $(b)$ holds for a given section $s: \mathcal{U} \rightarrow P$, then it holds for any other section on the same open set $\mathcal{U}$.

The proof of Theorem 1.1 is simple. The necessity is a consequence of the fact that, if there is the required immersion $f: \mathcal{U} \subset M \rightarrow \widetilde{M}$, one can pullback the Maurer-Cartan form $\varpi$ of $\widetilde{H}$ on $M$ and obtain a 1-form $\omega$ that satisfies (1.3) and (1.4). The sufficiency is obtained by an appropriate use of Frobenius' Theorem (see Sect. 2).

Theorem 1.1 gives an unified approach for determining complete sets of necessary and sufficient conditions for the existence of local immersions into homogeneous spaces. To have a flavor of the meaning of Eqs. (1.3) and (1.4), consider the case where there is a reductive decomposition $\widetilde{\mathfrak{h}}=\widetilde{\mathfrak{k}}+\widetilde{\mathfrak{m}}$ and two decompositions $\widetilde{\mathfrak{k}}=\mathfrak{g}+\mathfrak{g}^{\perp}$ and $\widetilde{\mathfrak{m}}=\mathfrak{m}+\mathfrak{m}^{\perp}$ into the direct sum of modules for the adjoint action of $\mathfrak{g}=\operatorname{Li} e(G)$. In this case, the $\widetilde{\mathfrak{h}}$-valued 1 -form $\omega$ has the form

$$
\omega=\omega^{\mathfrak{g}}+\omega^{\mathfrak{g}^{\perp}}+\omega^{\mathfrak{m}}+\omega^{\mathfrak{m}^{\perp}}
$$

and condition (1.3) means that $\omega^{\mathfrak{m}}$ coincides with $s^{*} \vartheta$ while $\omega^{\mathfrak{m}}{ }^{\perp}$ vanishes. At the same time, condition (1.4) corresponds to the existence of a 1-form $\omega^{\widetilde{\mathfrak{k}}}=\omega^{\mathfrak{g}}+\omega^{\mathfrak{g}^{\perp}}$ satisfying the system of equations

$$
\begin{aligned}
& {\left[\omega^{\mathfrak{g}}, \omega^{\mathfrak{m}}\right]+\left[\omega^{\mathfrak{g}^{\perp}}, \omega^{\mathfrak{m}}\right]=-d \omega^{\mathfrak{m}}-\frac{1}{2}\left[\omega^{\mathfrak{m}}, \omega^{\mathfrak{m}}\right] \widetilde{\mathfrak{m}},} \\
& d \omega^{\mathfrak{g}}+d \omega^{\mathfrak{g}^{\perp}}+\frac{1}{2}\left[\omega^{\mathfrak{g}}, \omega^{\mathfrak{g}}\right]+\left[\omega^{\mathfrak{g}}, \omega^{\mathfrak{g}^{\perp}}\right]+\frac{1}{2}\left[\omega^{\mathfrak{g}^{\perp}}, \omega^{\mathfrak{g}^{\perp}}\right]=-\frac{1}{2}\left[\omega^{\mathfrak{m}}, \omega^{\mathfrak{m}}\right]_{\mathfrak{\mathfrak { k }}}
\end{aligned}
$$

In the case of isometric immersions into spaces of constant curvature, these equations turn out to be equivalent to the classical Gauss-Codazzi-Ricci equations and the property that they are the only obstructions to be satisfied is a direct consequence of Theorem 1.1 and of its main corollaries (see Sect. 5.1).

In general, Eq. (1.4) is quite involved. But, if $\widetilde{\mathfrak{h}}=\sum_{p=-1}^{k-1} \widetilde{\mathfrak{h}}^{p}$ is a (quasi-)graded Lie algebra of depth 1 , it splits into a sequence of equations that can be solved with an iterative process (see Sects. 3.1 and 3.2). Further, if $\widetilde{P}$ is integrable and of finite type, in analogy with the results in [7], there is a sequence of locally defined maps on $\mathcal{U} \subset M$ which take values in appropriate cohomology groups and vanish if and only if condition (b) of Theorem 1.1 is 
satisfied, i.e., if and only if the generalized integrability problem is solvable (see Theorem 3.4). These maps are called essential $(G, \widetilde{G})$-curvatures, and they generalize, for instance, the classical Riemannian curvature in the integrability problem of Riemannian metrics. Under appropriate cohomological conditions, the essential $(G, \widetilde{G})$-curvatures are maps which are globally well defined on the total space of $\pi: P \rightarrow M$ and $G_{\sharp}$-equivariant (see Proposition 4.2 and Theorem 4.3).

Several applications of the general theory of $\widetilde{G}$-structures $\widetilde{\pi}: \widetilde{P} \rightarrow \widetilde{M}$ and the $G$ structures $\pi: P \rightarrow M$ induced on $\widetilde{P}$-regular submanifolds $M \subset \widetilde{M}$ are considered in Sect. 5 . There we first obtain in a unified fashion the classical Gauss-Codazzi-Ricci equations for the isometric immersions of a Riemannian manifold $(M, g)$ into Euclidean spaces, spheres, and hyperbolic spaces. Then, we consider the case of local conformal immersions of $(M, g)$ into spheres and get a new proof for Akivis' theorem on the existence of such immersions $([1,2])$. Additional new results on this topic are also given. Finally, we show how the general results yield the classical result by Andreotti and Hill on the existence of CR local embeddings of CR manifolds in $\mathbb{C}^{\widetilde{n}}$ ([5]), and we discuss the theorem on the existence of local CR quaternionic embeddings of an almost CR quaternionic manifold in $\mathbb{H} \mathrm{P}^{\tilde{n}}$ that we prove in [18].

The paper is organized as follows. In Sect. 2, we give the basic definitions and properties of homogeneous $\widetilde{G}$-structures and induced $G$-structures and prove Theorem 1.1. In Sect. 3 , we consider the case of (quasi-)graded Lie algebras, describe the above-mentioned iterative process, and prove Theorem 3.4, the main result of this section. In Sects. 4 and 5, we restrict to local immersions in flat 2 -reductive $\widetilde{G}$-structures and present the previously mentioned examples of applications.

Before concluding, we have to mention that a general study of the generalized integrability problem was also done by Rosly and Schwarz in [16]. In fact, this paper was strongly inspired by that one. There, the authors considered immersions into integrable $\widetilde{G}$-structures of finite type, established Theorem 1.1, and determined a finite sequence of obstructions. Main novelties of this paper with respect to [16] are the proof of Theorem 1.1 in coordinate-free language, which allows immediate extensions to the case of local immersions into homogeneous manifolds, and the introduction of a new sequence of cohomology groups and the corresponding essential curvatures. Note that this new setting can also be easily adapted to deal with other kinds of $G$-structures, as for instance those of higher order (see [19]). We also remark that the essential curvatures considered in this paper are in general different from Rosly and Schwarz' curvatures and actually determine a set of obstructions which completes the one presented in [16] (see Remark 5.2).

\section{$2 G$-structures immersible into homogeneous $\widetilde{G}$-structures}

\subsection{Homogeneous $G$-structures}

Let $M=H / K$ be an $n$-dimensional homogeneous manifold with an effective action. We throughout assume that $H$ is connected and simply connected and $K$ connected. For any $h \in H$, we denote by $L_{h} \in \operatorname{Diff}(M)$ the corresponding left action.

Given a frame $u_{o}=\left(e_{i}\right) \in L(M)$ at $o=e K$, let

$$
P=H \cdot u_{o}=\left\{h \cdot u_{o}:=\left(L_{h *} e_{i}\right), h \in H\right\} \subset L(M)
$$

be the $H$-orbit of $u_{o}$. One gets in this way a reduction $\pi: P \rightarrow M$ of $L(M)$, which we call the homogeneous $G$-structure associated with $M=H / K$. 
Note that if $K_{1}$ is the kernel of the isotropy representation

$$
K \rightarrow \mathrm{GL}\left(T_{o} M\right),\left.\quad k \mapsto L_{k *}\right|_{o},
$$

then the map $h \mapsto h \cdot u_{o}$ determines a natural bundle isomorphism between $H / K_{1} \rightarrow M=$ $H / K$ and the $G$-structure $\pi: P \rightarrow M$, with $G \simeq K / K_{1}$.

From now on, we assume a fixed choice of $u_{o}$ and tacitly use it to identify $T_{o} M$ with $\mathbb{R}^{n}$ and $\pi: P \rightarrow M$ with $H / K_{1} \rightarrow H / K$. Then, if we consider the canonical projections

$$
\pi_{1}: H \rightarrow P=H / K_{1}, \quad p: \mathfrak{h} \rightarrow \mathfrak{h} / \mathfrak{k} \simeq T_{o} M,
$$

the soldering form $\vartheta$ of $P$ can be determined from the Maurer-Cartan form $\varpi$ of $H$ as the unique $\mathbb{R}^{n}$-valued 1 -form which satisfies

$$
\pi_{1}^{*} \vartheta=p \circ \varpi .
$$

In the following, we define homogeneous $G$-structure any pair $(H / K, P)$ consisting of an homogeneous manifold $M=H / K$ and a fixed associated homogeneous $G$-structure $P$. Further, an homogeneous $G$-structure $\left(H^{\prime} / K^{\prime}, P^{\prime}\right)$ is defined extension of $(H / K, P)$ if $H^{\prime} / K^{\prime}$ is locally diffeomorphic to $H / K, P^{\prime}$ locally equivalent to $P$ as a $G$-structure, and $\mathfrak{h}=\operatorname{Lie}(H)$ is a proper subalgebra of $\mathfrak{h}^{\prime}=\operatorname{Lie}\left(H^{\prime}\right)$ with $\mathfrak{k}=\mathfrak{h} \cap \mathfrak{k}^{\prime}$. The homogeneous $G$-structure $(H / K, P)$ is inextendible if it does not admit any extension.

As mentioned in the introduction, Theorem 1.1 fully characterizes immersible $G$ structures. Before the proof, we give a simple characterization of $\widetilde{P}$-regular submanifolds, which hold for any (not necessarily homogeneous) $\widetilde{G}$-structure $\widetilde{\pi}: \widetilde{P} \rightarrow \widetilde{M}$.

Lemma 2.1 A submanifold $M \subset \widetilde{M}$ is $\widetilde{P}$-regular if and only if for any $x \in M$ there exists an adapted frame $u$ at $x$. In this case, the principal bundle $\pi_{\sharp}: P_{\sharp} \rightarrow M$ is a $G_{\sharp}$-reduction of $\left.\widetilde{P}\right|_{M}$ with $G_{\sharp}$ as in (1.1).

This lemma is proved by first checking the existence of local smooth sections of $\left.\widetilde{P}\right|_{M}$ taking values in $P_{\sharp}$ and then applying Lemma 1 in [11, p.84]. We omit the details for the sake of brevity.

\subsection{Proof of Theorem 1.1}

Let $f: \mathcal{U} \subset M \rightarrow \widetilde{M}$ be a $(P, \widetilde{P})$-regular embedding, which we use to identify $\mathcal{U}$ with a submanifold of $\widetilde{M}$ and $\left.P\right|_{\mathcal{U}}$ with the induced $G$-structure. Without loss of generality, we may assume $\mathcal{U}$ to be contractible, consider a local section $s_{\sharp}: \mathcal{U} \rightarrow P_{\sharp}$ of the subbundle $\left.P_{\sharp} \subset \widetilde{P}\right|_{\mathcal{U}}$ and the corresponding section $s: \mathcal{U} \rightarrow P$ of $P$ induced via the identification $P \simeq P_{\sharp} / N_{\sharp}$. By local triviality of the fibration $\tilde{\pi}_{1}: \widetilde{H} \rightarrow \widetilde{P}=\widetilde{H} / \widetilde{K}_{1}$, we may also pick a local section $\widetilde{s}:\left.\mathcal{U} \rightarrow \widetilde{H}\right|_{\mathcal{U}}$ satisfying $\tilde{\pi}_{1} \circ \widetilde{s}=s_{\sharp}$. The situation is summarized by the following diagram:

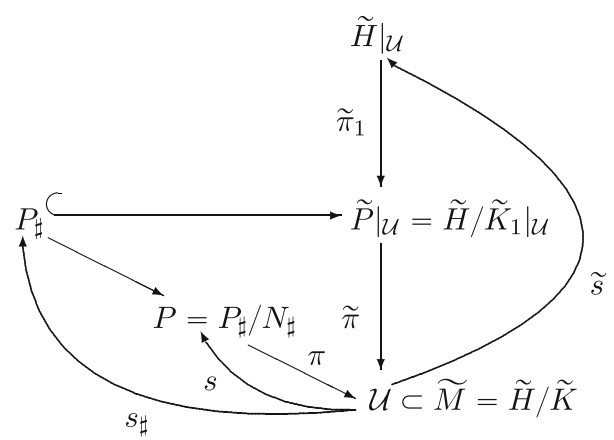


Let $\omega=\widetilde{s}^{*} \varpi$, where $\varpi$ is the Maurer-Cartan form of $\widetilde{H}$. Then, Eq. (1.3) is checked observing that

$$
\widetilde{p} \circ \omega=\widetilde{s}^{*}(\widetilde{p} \circ \varpi)=\widetilde{s}^{*}\left(\widetilde{\pi}_{1}^{*} \widetilde{\vartheta}\right)=s_{\sharp}^{*} \widetilde{\vartheta}=\left(s^{*} \vartheta^{1}, \ldots, s^{*} \vartheta^{n}, 0, \ldots, 0\right),
$$

where the second equality follows from (2.1) applied to the soldering form $\widetilde{\vartheta}$ of $\widetilde{P}$ and the last equality is a direct consequence of definitions of soldering 1-forms and induced $G$-structures. The Eq. (1.4) follows from the Maurer-Cartan equation of $\varpi$.

Conversely, let $\omega$ be a 1-form satisfying (1.3), (1.4) and consider the 1-form $\omega-\varpi$ on the product $\mathcal{U} \times \widetilde{H}$ with the associated distribution $\mathcal{D} \subset T(\mathcal{U} \times \widetilde{H})$ of rank $n$, defined by

$$
\mathcal{D}=\cup_{y \in \mathcal{U} \times \widetilde{H}} \mathcal{D}_{y}, \quad \mathcal{D}_{y}=\left.\operatorname{Ker}(\omega-\varpi)\right|_{y} .
$$

Using (1.4), one checks that $\mathcal{D}$ is involutive, hence integrable by Frobenius' Theorem. For a chosen $x \in \mathcal{U}$, an integral leaf through the point $(x, e)$ is the graph of a unique local map $\widetilde{s}: \mathcal{U} \rightarrow \widetilde{H}$ with $\omega=\widetilde{s}^{*} \varpi$ and $\widetilde{s}(x)=e$.

We now claim that, by possibly taking a smaller $\mathcal{U}$, the map

$$
f=\tilde{\pi} \circ \tilde{\pi}_{1} \circ \tilde{s}: \mathcal{U} \subset M \rightarrow \widetilde{M}
$$

is a $(P, \widetilde{P})$-regular embedding. Using (1.3), one sees that $\left.f_{*}\right|_{x}$ is injective so that $f$ is a local embedding around $x$. Moreover, given the section $s_{\sharp}:=\widetilde{\pi}_{1} \circ \widetilde{s}:\left.\mathcal{U} \rightarrow \widetilde{P}\right|_{\mathcal{U}}$ of $\left.\widetilde{P}\right|_{\mathcal{U}}$, we may consider the $G_{\sharp}$-reduction

$$
P_{\sharp}=\left.\left\{s_{\sharp}(x) \cdot \widetilde{A}: x \in \mathcal{U}, \widetilde{A} \in G_{\sharp}\right\} \subset \widetilde{P}\right|_{\mathcal{U}} .
$$

By (2.1) and (1.3), we get

$$
s_{\sharp}^{*} \widetilde{\vartheta}=\widetilde{s}^{*} \tilde{\pi}_{1}^{*} \widetilde{\vartheta}=\tilde{p} \circ\left(\widetilde{s}^{*} \varpi\right)=\tilde{p} \circ \omega=\left(s^{*} \vartheta^{1}, \ldots, s^{*} \vartheta^{n}, 0, \ldots, 0\right) .
$$

This relation between the soldering forms $\widetilde{\vartheta}, \vartheta$ of $\widetilde{P}$ and $P$, respectively, says that $f$ is $(P, \widetilde{P})$-regular with bundle of adapted frames isomorphic with $P_{\sharp}$.

Since we just proved that the existence of solutions to (1.3) and (1.4) for a given section $s: \mathcal{U} \rightarrow P$ is equivalent to local immersibility of $P$ (a property clearly independent on the choice of $s$ ), the last claim of the theorem follows immediately.

Remark 2.2 Let $\left(\widetilde{H}^{\prime} / \widetilde{K}^{\prime}, \widetilde{P}^{\prime}\right)$ be a proper extension of the homogeneous $\widetilde{G}$-structure $(\widetilde{H} / \widetilde{K}, \widetilde{P})$. Since any $\widetilde{\mathfrak{h}}$-valued 1 -form is an $\widetilde{\mathfrak{h}}^{\prime}$-valued 1 -form satisfying additional conditions, the Eqs. (1.3) and (1.4) on $\widetilde{\mathfrak{h}}$-valued 1-forms are equivalent to the system on $\widetilde{\mathfrak{h}}^{\prime}$-valued 1-forms given by (1.3), (1.4) and such constraints. On the other hand, since $\widetilde{P}^{\prime} \simeq \widetilde{P}$, Theorem 1.1 implies that there is an $\widetilde{\mathfrak{h}}$-valued solution of (1.3) and (1.4) if and only if there is an $\widetilde{\mathfrak{h}}^{\prime}$-valued solution. Due to this, inextendible homogeneous $\widetilde{G}$-structures are more appropriate than the extendible ones for determining minimal sets of obstructions for a given generalized integrability problem. 


\section{$3 k$-reductive $\widetilde{G}$-structures and $(G, \widetilde{G})$-curvatures}

\section{$3.1 k$-reductive $G$-structures}

We recall that a Lie algebra $\mathfrak{h}$ is a graded Lie algebra of depth 1 and height $k$ (shortly, graded Lie algebra of height $k$ ) if it admits a direct sum decomposition of vector spaces

$$
\mathfrak{h}=\sum_{p=-1}^{k-1} \mathfrak{h}^{p}
$$

such that

$$
\left[\mathfrak{h}^{r}, \mathfrak{h}^{s}\right] \subset \mathfrak{h}^{r+s} \quad \text { for all } r, s,
$$

where $\mathfrak{h}^{r+s}=\{0\}$ for $r+s \notin\{-1,0, \ldots, k-1\}$. If a Lie algebra $\mathfrak{h}$ admits a vector space decomposition (3.1) and satisfies (3.2) only when $r+s \geq-1$, we call it quasi-graded of height $k$.

Note that any Lie algebra $\mathfrak{g}$ can be considered as a quasi-graded Lie algebra of height $k=0$, while the quasi-graded Lie algebras of height $k=1$ coincide with the Lie algebras endowed with a reductive decomposition.

The $n$-dimensional homogeneous spaces $M=H / K$ with an effective action, quasi-graded Lie algebra $\mathfrak{h}=\operatorname{Lie}(H)$ of height $k$, and isotropy subalgebra

$$
\mathfrak{k}=\operatorname{Lie}(K)=\sum_{p=0}^{k-1} \mathfrak{h}^{p}
$$

are called $k$-reductive; they have been considered by I. L. Kantor $([4,8])$ as natural generalizations of reductive homogeneous spaces. In this case,

$$
\begin{aligned}
& \operatorname{dim} \mathfrak{h}^{-1}=\operatorname{dim}(\mathfrak{h} / \mathfrak{k})=n \quad \text { and } \\
& \text { for any } X \in \mathfrak{h}^{p}, p \geq 0, \quad\left[X, \mathfrak{h}^{-1}\right]=0 \quad \text { implies } \quad X=0,
\end{aligned}
$$

by transitivity and effectiveness of the action of $H$ on $M$.

We call $k$-reductive $G$-structure any homogeneous $G$-structure $(H / K, P)$ associated with a $k$-reductive space $H / K$. Under an identification $\mathfrak{h} / \mathfrak{k} \simeq \mathbb{R}^{n}$ given by a given frame $u_{o}$ of $\left.P\right|_{o}$, the Lie algebra $\mathfrak{g} \subset \mathfrak{g l}_{n}(\mathbb{R})$ of the structure group $G$ of $P$ is identifiable with $\mathfrak{h}^{0}$.

When $\mathfrak{h}$ is a graded Lie algebra, it is known that $(H / K, P)$ is locally identifiable with the flat $G$-structure on $\mathbb{R}^{n}$, i.e., it is locally integrable. We call it flat $k$-reductive $G$-structure. Its Lie algebra $\mathfrak{h}=\sum_{p=-1}^{k-1} \mathfrak{h}^{p}$ is a subalgebra of the maximal prolongation of $\mathfrak{h}^{0} \subset \mathfrak{g l}_{n}(\mathbb{R})$, defined as follows.

Definition 3.1 The maximal transitive prolongation of a subalgebra $\mathfrak{h}^{0}$ of $\mathfrak{g l}_{n}(\mathbb{R})$ is the maximal graded Lie algebra

$$
\mathfrak{h}_{\infty}=\sum_{p=-1}^{\infty} \mathfrak{h}_{\infty}^{p},
$$

with $\mathfrak{h}_{\infty}^{-1}=\mathbb{R}^{n}, \mathfrak{h}_{\infty}^{0}=\mathfrak{h}^{0}$ and satisfying the following two properties:

1. The adjoint action of $\mathfrak{h}_{\infty}^{0}$ on $\mathfrak{h}_{\infty}^{-1}$ is the standard action of $\mathfrak{h}^{0}$ on $\mathbb{R}^{n}$,

2. For any $X \in \mathfrak{h}_{\infty}^{p}, p \geq 0$, if $\left[X, \mathfrak{h}_{\infty}^{-1}\right]=0$, then $X=0$. 
It is known that the maximal prolongation $\mathfrak{h}_{\infty}$ is uniquely determined by the algebra $\mathfrak{h}^{-1}+\mathfrak{h}^{0} \subset \mathbb{R}^{n}+\mathfrak{g l}_{n}(\mathbb{R})$ and it might be infinite dimensional. When it is finite dimensional, the Lie algebra $\mathfrak{g}=\mathfrak{h}^{0}$ is called of finite type. Accordingly, also the $G$-structures with Lie algebras $\operatorname{Lie}(G)=\mathfrak{g}$ are called of finite type (for further details, see, e.g., [9, Ch.1]).

A flat $k$-reductive $G$-structure $(H / K, P)$, with $P$ of finite type and $\mathfrak{h}$ equal to its maximal prolongation $\mathfrak{h}=\mathfrak{h}_{\infty}=\sum_{p=-1}^{k-1} \mathfrak{h}_{\infty}^{p}$, is inextendible (this follows from Sternberg prolongation theory of bundles, see [22]). Further, one can check that the following holds (see [8, Thms. 17-18]): if an homogeneous space $H^{\prime} / K^{\prime}$ admits an invariant Cartan connection modeled on $H / K$ and is of maximal dimension (that is $\operatorname{dim} H^{\prime}=\operatorname{dim} H$ ) then:

- $H^{\prime} / K^{\prime}$ is $k$-reductive,

- Lie $\left(H^{\prime}\right)$ is a quasi-graded Lie algebra with underlying graded vector space isomorphic to $\mathfrak{h}$,

$-\operatorname{Lie}\left(K^{\prime}\right) \simeq \operatorname{Lie}(K)=\sum_{p=0}^{k-1} \mathfrak{h}_{\infty}^{p}$.

First examples of such homogeneous spaces and their associated $k$-reductive $G$-structures $\left(H^{\prime} / K^{\prime}, P^{\prime}\right)$ are the three simply connected Riemannian manifolds of constant curvature and their orthonormal frame bundles (see Sect. 5.1).

\subsection{Immersible $G$-structures of order $p$ and associated $(G, \tilde{G})$-curvatures}

Let $(\widetilde{H} / \widetilde{K}, \widetilde{P})$ be a $k$-reductive $\widetilde{G}$-structure on an $\widetilde{n}$-dimensional homogeneous manifold $\widetilde{M}=\widetilde{H} / \widetilde{K}$ and $\pi: P \rightarrow M$ a $G$-structure on an $n$-dimensional manifold $M$ with soldering form $\vartheta=\left(\vartheta^{1}, \ldots, \vartheta^{n}\right)$, where $G$ is isomorphic to $G_{\sharp} / N_{\sharp}$ with $G_{\sharp}, N_{\sharp} \subset \widetilde{G}$ as in (1.1) and (1.2).

Combining Theorem 1.1 with the decomposition $\widetilde{\mathfrak{h}}=\sum_{p=-1}^{k-1} \widetilde{\mathfrak{h}}^{p}$, one gets that $P$ is locally immersible in $\widetilde{P}$ if and only if, for any local section $s: \mathcal{U} \subset M \rightarrow P$ of $P$, there exist $k+11$-forms

$$
\omega^{p}: T \mathcal{U} \rightarrow \widetilde{\mathfrak{h}}^{p}, \quad-1 \leq p \leq k-1,
$$

with

$$
\omega^{-1}=\left(s^{*} \vartheta^{1}, \ldots, s^{*} \vartheta^{n}, 0, \ldots, 0\right)
$$

and the others satisfying the system of $k+1$ equations

$$
\begin{aligned}
& d \omega^{p-1}+\frac{1}{2} \sum_{r=0}^{p-1}\left[\omega^{r}, \omega^{p-1-r}\right]+\frac{1}{2}\left[\omega^{-1}, \omega^{-1}\right]^{\widetilde{\mathfrak{h}}^{p-1}}=-\left[\omega^{-1}, \omega^{p}\right], \quad 0 \leq p \leq k-1 \\
& d \omega^{k-1}+\frac{1}{2} \sum_{r=0}^{k-1}\left[\omega^{r}, \omega^{k-1-r}\right]+\frac{1}{2}\left[\omega^{-1}, \omega^{-1}\right]^{\widetilde{\mathfrak{h}}^{k-1}}=0
\end{aligned}
$$

where we denoted by $(\cdot)^{\widetilde{\mathfrak{h}}^{r}}$ the natural projection onto the subspace $\widetilde{\mathfrak{h}}^{r}$ of $\widetilde{\mathfrak{h}}$.

We stress the fact that if (3.3) and (3.4) hold for a choice of $s$, then they hold for any other section of $P$ on the same open set $\mathcal{U}$. The very same property is also shared by the subsystem of equations which consists of (3.3) and just the first $p$ equations of (3.4) for some $p<k+1$. Due to this, in order to simplify the quest for solutions, it is always advisable to make a choice for the section $s$ (from which the associated 1-form $\omega^{-1}$ is always uniquely determined by (3.3)) that makes the explicit expression of (3.3) as simple as possible (see, for instance, Sect. 5.2).

All this motivates the following: 
Definition 3.2 The $G$-structure $P$ is defined locally immersibile into $(\widetilde{H} / \widetilde{K}, \widetilde{P})$ to order $p$ if, around any point, it admits a local section $s$ and forms $\omega^{-1}, \ldots, \omega^{p-1}$ which satisfy (3.3) and the first $p$ equations of (3.4). Any $p+1$-tuple $\left(s, \omega^{0}, \ldots, \omega^{p-1}\right)$ as above is called admissible.

Let $P$ be immersible to order $p$ and $\left(s, \omega^{0}, \ldots, \omega^{p-1}\right)$ an admissible tuple. Let also $V=\mathbb{R}^{\tilde{n}} \simeq \widetilde{\mathfrak{h}}^{-1}$ and denote by $W \simeq \mathbb{R}^{n}$ the $n$-dimensional subspace of $V$ which corresponds to the vanishing of the last $\tilde{n}-n$ standard coordinates of $\mathbb{R}^{\widetilde{n}}$. For any integer $r=0,1, \ldots, p$, we define annihilator of level $r$ in $\widetilde{\mathfrak{h}}^{p-1}$ the subspace of $\widetilde{\mathfrak{h}}^{p-1}$ given by

$$
\mathfrak{c}_{r}^{p-1}=\left\{X \in \widetilde{\mathfrak{h}}^{p-1} \mid\left[\ldots\left[\left[X, w_{1}\right], w_{2}\right] \ldots, w_{r}\right]=0 \text { for all } w_{1}, \ldots, w_{r} \in W\right\} .
$$

For instance $\mathfrak{c}_{0}^{p-1}=0$ and $\mathfrak{c}_{1}^{p-1}$ is the centralizer of $W$ in $\widetilde{\mathfrak{h}}^{p-1}$. We also set $\mathfrak{c}_{p+1}^{p-1}:=\widetilde{\mathfrak{h}}^{p-1}$ and consider the increasing filtration of $\widetilde{\mathfrak{h}}^{p-1}$ given by

$$
0=\mathfrak{c}_{0}^{p-1} \subset \mathfrak{c}_{1}^{p-1} \subset \cdots \subset \mathfrak{c}_{r}^{p-1} \subset \cdots \subset \mathfrak{c}_{p}^{p-1} \subset \mathfrak{c}_{p+1}^{p-1}=\widetilde{\mathfrak{h}}^{p-1} .
$$

Let $p_{r}^{\prime}: \widetilde{\mathfrak{h}}^{p-1} \rightarrow \widetilde{\mathfrak{h}}^{p-1} / \mathfrak{c}_{r}^{p-1}$ be the natural projection. We define $(G, \widetilde{G})$-curvature of level $r$ and order $p+1$ associated with $\left(s, \omega^{0}, \ldots, \omega^{p-1}\right)$ the $\widetilde{\mathfrak{h}}^{p-1} / \mathfrak{c}_{r}^{p-1}$-valued 2-form on the domain of $s$ given by

$$
\widehat{\Omega}_{r}^{p-1}=p_{r}^{\prime} \circ\left(d \omega^{p-1}+\frac{1}{2} \sum_{s=0}^{p-1}\left[\omega^{s}, \omega^{p-1-s}\right]+\frac{1}{2}\left[\omega^{-1}, \omega^{-1}\right]^{\widetilde{\mathfrak{h}}^{p-1}}\right) .
$$

For any $r=0,1, \ldots, p$, we fix a subspace $\mathfrak{c}_{r}^{p-1 \perp}$ complementary to $\mathfrak{c}_{r}^{p-1}$ in $\mathfrak{c}_{r+1}^{p-1}$. One gets in this way a vector space direct sum decomposition

$$
\widetilde{\mathfrak{h}}^{p-1}=\bigoplus_{s=0}^{p} \mathfrak{c}_{s}^{p-1 \perp}, \quad \text { where } \quad \mathfrak{c}_{r}^{p-1}=\bigoplus_{s=0}^{r-1} \mathfrak{c}_{s}^{p-1 \perp},
$$

which determines a corresponding projection $p_{r}^{\prime \prime}: \widetilde{\mathfrak{h}}^{p-1} \rightarrow \mathfrak{c}_{r}^{p-1 \perp}$. We define complementary $(G, \widetilde{G})$-curvature of level $r$ and order $p+1$ associated with $\left(s, \omega^{0}, \ldots, \omega^{p-1}\right)$ and the decomposition (3.6) the $\mathfrak{c}_{r}^{p-1 \perp}$-valued 2-form on the domain of $s$ given by

$$
\widetilde{\Omega}_{r}^{p-1}=p_{r}^{\prime \prime} \circ\left(d \omega^{p-1}+\frac{1}{2} \sum_{s=0}^{p-1}\left[\omega^{s}, \omega^{p-1-s}\right]+\frac{1}{2}\left[\omega^{-1}, \omega^{-1}\right]^{\widetilde{\mathfrak{h}}^{p-1}}\right) .
$$

Finally, we define total $(G, \widetilde{G})$-curvature of order $p+1$ associated with $\left(s, \omega^{0}, \ldots, \omega^{p-1}\right)$ the $\widetilde{\mathfrak{h}}^{p-1}$-valued 2 -form on the domain of $s$ given by

$$
\Omega^{p-1}=d \omega^{p-1}+\frac{1}{2} \sum_{r=0}^{p-1}\left[\omega^{r}, \omega^{p-1-r}\right]+\frac{1}{2}\left[\omega^{-1}, \omega^{-1}\right]^{\widetilde{\mathfrak{h}}^{p-1}} .
$$

We observe that for any fixed choice of a decomposition (3.6) as above, $\widetilde{\mathfrak{h}}^{p-1} / \mathfrak{c}_{r}^{p-1}$ is naturally identified with $\bigoplus_{s=r}^{p} \mathfrak{c}_{s}^{p-1 \perp}$, and the total curvature is the sum

$$
\Omega^{p-1}=\widehat{\Omega}_{r}^{p-1}+\sum_{s=0}^{r-1} \widetilde{\Omega}_{s}^{p-1}
$$


of the curvature of level $r$ and the complementary curvatures of lower levels.

The question whether a given $G$-structure $P$ is locally immersible in $\widetilde{P}$ is therefore solvable by the following iterative procedure. First, fix a section $s: \mathcal{U} \subset M \rightarrow P$ and set $\omega^{-1}=$ $\left(s^{*} \vartheta^{1}, \ldots, s^{*} \vartheta^{n}, 0, \ldots, 0\right)$. Secondly, look for an $\widetilde{\mathfrak{h}}^{0}$-valued 1 -form $\omega^{0}$ which solves the first equation of (3.4). Such equation is purely algebraic in $\omega^{0}$ and, using the total $(G, \widetilde{G})$ curvature $\Omega^{-1}$, can be written as

$$
\left[\omega^{-1}, \omega^{0}\right]=-\Omega^{-1} .
$$

If there exists a solution $\omega^{0}, P$ is immersible to order 1 . We may then compute the total $(G, \widetilde{G})$-curvature $\Omega^{0}$ and look for a $\widetilde{\mathfrak{h}}^{1}$-valued 1 -form $\omega^{1}$ solution to the next (algebraic) equation

$$
\left[\omega^{-1}, \omega^{1}\right]=-\Omega^{0},
$$

and so on. In the final step, we have that $P$ is immersible if and only if there exists a choice of an admissible $k+1$-tuple $\left(s, \omega^{0}, \ldots, \omega^{k-1}\right)$ whose associated total $(G, \widetilde{G})$-curvature $\Omega^{k-1}$ vanishes identically.

These operations show that, at the $p^{\text {th }}$-step, the immersibility to the higher order depends on an algebraic obstruction, corresponding to the pointwise solvability of the non-homogeneous linear equation

$$
\left[\omega^{-1}, \omega^{p}\right]=-\Omega^{p-1}
$$

in the unknown $\omega^{p}$. Moreover, using the $(G, \widetilde{G})$-curvatures of level $\mathrm{r}$ and the complementary curvatures, such equation splits for any $r=0,1, \ldots, p$ into the system of $r$ equations

$$
\begin{array}{ll}
p_{r}^{\prime} \circ\left[\omega^{-1}, \omega^{p}\right]=-\widehat{\Omega}_{r}^{p-1} & \text { and } \\
p_{s}^{\prime \prime} \circ\left[\omega^{-1}, \omega^{p}\right]=-\widetilde{\Omega}_{s}^{p-1} & \text { for any } s=0, \ldots, r-1 .
\end{array}
$$

We, respectively, call them equation of level $\mathrm{r}$ and complementary equations of levels $0, \ldots, r-1$. We remark that the equation of level $r$ depends just on $p_{r}^{\prime} \circ \omega^{p}$ and not on the entire 1-form $\omega^{p}$.

\subsection{Immersible $G$-structures and flat $k$-reductive $\widetilde{G}$-structures}

When $(\widetilde{H} / \widetilde{K}, \widetilde{P})$ is flat $k$-reductive, the algebraic conditions on the total $(G, \widetilde{G})$-curvature $\Omega^{p-1}$ and its components $\widehat{\Omega}_{r}^{p-1}$ of level $r$ can be expressed in cohomological terms. For this, we need the following.

Lemma 3.3 If $(\widetilde{H} / \widetilde{K}, \widetilde{P})$ is flat $k$-reductive, the total $(G, \widetilde{G})$-curvature associated with an admissible tuple $\left(s, \omega^{0}, \ldots, \omega^{p-1}\right)$ satisfies the generalized Bianchi identity $\left[\omega^{-1}, \Omega^{p-1}\right]=$ 0 . In particular, its component of level $r$ satisfies $p_{r}^{\prime} \circ\left[\omega^{-1}, \widehat{\Omega}_{r}^{p-1}\right]=0$.

Proof Since $\left(s, \omega^{0}, \ldots, \omega^{p-1}\right)$ is admissible and $\widetilde{\mathfrak{h}}^{-1}$ is abelian, one has

$$
d \omega^{r}+\frac{1}{2} \sum_{s=0}^{r}\left[\omega^{s}, \omega^{r-s}\right]=-\left[\omega^{-1}, \omega^{r+1}\right], \quad \text { for all }-1 \leq r \leq p-2 .
$$

Applying $d$ to both sides when $r=p-2$, one gets

$$
0=-d\left(d \omega^{p-2}\right)=\frac{1}{2} \sum_{r=0}^{p-2} d\left[\omega^{r}, \omega^{p-2-r}\right]+d\left[\omega^{-1}, \omega^{p-1}\right] .
$$


This equality together with admissibility of $\left(s, \omega^{0}, \ldots, \omega^{p-1}\right)$ yields

$$
\begin{aligned}
0 & =\frac{1}{2} \sum_{r=0}^{p-2}\left[d \omega^{r}, \omega^{p-2-r}\right]-\frac{1}{2} \sum_{r=0}^{p-2}\left[\omega^{r}, d \omega^{p-2-r}\right]+\left[d \omega^{-1}, \omega^{p-1}\right]-\left[\omega^{-1}, d \omega^{p-1}\right] \\
& =\sum_{r=-1}^{p-2}\left[d \omega^{r}, \omega^{p-2-r}\right]-\left[\omega^{-1}, d \omega^{p-1}\right] \\
& =-\frac{1}{2} \sum_{r=0}^{p-2} \sum_{s=0}^{r}\left[\left[\omega^{s}, \omega^{r-s}\right], \omega^{p-2-r}\right]-\sum_{r=-1}^{p-2}\left[\left[\omega^{-1}, \omega^{r+1}\right], \omega^{p-2-r}\right]-\left[\omega^{-1}, d \omega^{p-1}\right] .
\end{aligned}
$$

On the other hand, it is not difficult to see $\sum_{r=0}^{p-2} \sum_{s=0}^{r}\left[\left[\omega^{s}, \omega^{r-s}\right], \omega^{p-2-r}\right]=0$ and

$$
\begin{aligned}
\sum_{r=-1}^{p-2}\left[\left[\omega^{-1}, \omega^{r+1}\right], \omega^{p-2-r}\right] & =\frac{1}{2} \sum_{r=-1}^{p-2}\left[\omega^{-1},\left[\omega^{r+1}, \omega^{p-2-r}\right]\right] \\
& =\frac{1}{2} \sum_{s=0}^{p-1}\left[\omega^{-1},\left[\omega^{s}, \omega^{p-1-s}\right]\right],
\end{aligned}
$$

so that $0=-\frac{1}{2} \sum_{s=0}^{p-1}\left[\omega^{-1},\left[\omega^{s}, \omega^{p-1-s}\right]\right]-\left[\omega^{-1}, d \omega^{p-1}\right]=-\left[\omega^{-1}, \Omega^{p-1}\right]$.

It remains to prove the second part of the lemma. From $\left[\omega^{-1}, \Omega^{p-1}\right]=0$, one first infers $p_{r}^{\prime} \circ\left[\omega^{-1}, \Omega^{p-1}\right]=0$. Since $\operatorname{ad}_{w}\left(\mathfrak{c}_{r}^{p-1}\right) \subset \mathfrak{c}_{r-1}^{p-2} \subset \mathfrak{c}_{r}^{p-2}$ for all $w \in W$, the claim follows directly from the fact that $\omega^{-1}$ is $W$-valued.

This lemma suggests to consider the following differential complex. For any nonnegative integers $r, p$, and $q$, we set

$$
C_{r}^{0, q}(\widetilde{\mathfrak{h}}, W):=V \otimes \Lambda^{q} W^{*}, \quad C_{r}^{p+1, q}(\widetilde{\mathfrak{h}}, W):=\left(\widetilde{\mathfrak{h}}^{p} / \mathfrak{c}_{r}^{p}\right) \otimes \Lambda^{q} W^{*} .
$$

Consider the generalized Spencer operator $\partial: C_{r}^{p, q}(\widetilde{\mathfrak{h}}, W) \rightarrow C_{r}^{p-1, q+1}(\widetilde{\mathfrak{h}}, W)$ given by

$$
\partial c\left(w_{1}, \ldots, w_{q+1}\right):=\sum_{i=1}^{q+1}(-1)^{i} p_{r}^{\prime} \circ\left[c\left(w_{1}, \ldots, w_{i-1}, \hat{w}_{i}, w_{i+1}, \ldots, w_{q+1}\right), w_{i}\right]
$$

for any $c \in C_{r}^{p, q}(\widetilde{\mathfrak{h}}, W)$ and $w_{1}, \ldots, w_{q+1} \in W$. One can directly check that $\partial^{2}=0$ and consider the corresponding differential complex

$$
\cdots \stackrel{\partial}{\longrightarrow} C_{r}^{p+1, q-1}(\widetilde{\mathfrak{h}}, W) \stackrel{\partial}{\longrightarrow} C_{r}^{p, q}(\widetilde{\mathfrak{h}}, W) \stackrel{\partial}{\longrightarrow} C_{r}^{p-1, q+1}(\widetilde{\mathfrak{h}}, W) \stackrel{\partial}{\longrightarrow} \cdots .
$$

Let $Z_{r}^{p, q}(\widetilde{\mathfrak{h}}, W)$ and $B_{r}^{p, q}(\widetilde{\mathfrak{h}}, W)$ be the spaces of $(p, q)$-cocycles and $(p, q)$-coboundaries of this complex and set

$$
H_{r}^{p, q}(\widetilde{\mathfrak{h}}, W)=Z_{r}^{p, q}(\widetilde{\mathfrak{h}}, W) / B_{r}^{p, q}(\widetilde{\mathfrak{h}}, W) .
$$

We call it $(p, q)$-cohomology group of level $r$.

The following theorem extends Guillemin's results on the integrability problem for $G$ structures (see [7]) to the generalized integrability problem. 
Theorem 3.4 Let $(\widetilde{H} / \widetilde{K}, \widetilde{P})$ be a flat $k$-reductive $\widetilde{G}$-structure on an homogeneous manifold $\widetilde{M}=\widetilde{H} / \widetilde{K}$ of dimension $\widetilde{n}$ and $\pi: P \rightarrow M$ a G-structure on a manifold $M$ of dimension $n$ with $G$ isomorphic to $G_{\sharp} / N_{\sharp}$, where $G_{\sharp}, N_{\sharp} \subset \widetilde{G}$ are as in (1.1) and (1.2).

Assume also that $P$ is locally immersible in $\widetilde{P}$ to order $p$ and, for any admissible tuple $\left(s, \omega^{0}, \ldots, \omega^{p-1}\right)$ on some open set $\mathcal{U} \subset M$, use the frames $s_{x}, x \in \mathcal{U}$, to identify the tangent spaces $T_{x} M$ with $W$. Then,

i) for any admissible $\left(s, \omega^{0}, \ldots, \omega^{p-1}\right)$, the associated total $(G, \widetilde{G})$-curvature tensors $\left.\Omega^{p-1}\right|_{x} \in \widetilde{\mathfrak{h}}^{p-1} \otimes \Lambda^{2} T_{x}^{*} M \simeq C^{p, 2}(\widetilde{\mathfrak{h}}, W)$ satisfy

$$
\partial\left(\left.\Omega^{p-1}\right|_{x}\right)=0
$$

and $\partial\left(\left.\widehat{\Omega}_{r}^{p-1}\right|_{x}\right)=0$ for all projections $\left.\widehat{\Omega}_{r}^{p-1}\right|_{x} \in C_{r}^{p, 2}(\widetilde{\mathfrak{h}}, W), 1 \leq r \leq p$;

ii) $P$ is locally immersible in $\widetilde{P}$ to order $p+1$ if and only if, around any point $x_{o}$, there exists an admissible tuple $\left(s, \omega^{0}, \ldots, \omega^{p-1}\right)$ such that $\left[\left.\Omega^{p-1}\right|_{x}\right] \in H^{p, 2}(\widetilde{\mathfrak{h}}, W)$ is zero at any $x$ where it is defined.

Proof (i) We first observe that given an admissible tuple $\left(s, \omega^{0}, \ldots, \omega^{p-1}\right)$ on $\mathcal{U} \subset M$, any local $\widetilde{\mathfrak{h}}^{s}$-valued $q$-form is naturally identified with a map from $\mathcal{U}$ to $C^{s-1, q}(\widetilde{\mathfrak{h}}, W)$. The Eq. (3.8) is then a consequence of definitions, Lemma 3.3 and the fact that $\omega^{-1}=$ $\left(s^{*} \vartheta^{1}, \ldots, s^{*} \vartheta^{n}, 0, \ldots, 0\right)$ while the last claim follows from the fact that the natural projection from $C^{p, q}(\widetilde{\mathfrak{h}}, W)$ to $C_{r}^{p, q}(\widetilde{\mathfrak{h}}, W)$ is a morphism of differential complexes.

(ii) We recall that $P$ is locally immersible in $\widetilde{P}$ to order $p+1$ if and only if, around any point $x_{o}$, there is an admissible tuple $\left(s, \omega^{0}, \ldots, \omega^{p-1}\right)$ with associated total $(G, \widetilde{G})$-curvature $\Omega^{p-1}$ and a smooth map $\omega^{p}: \mathcal{U} \rightarrow C^{p+1,1}(\widetilde{\mathfrak{h}}, W)$ such that $\partial \omega^{p}=-\Omega^{p-1}$.

If this is the case, the class $\left[\left.\Omega^{p-1}\right|_{x}\right] \in H^{p, 2}(\widetilde{\mathfrak{h}}, W)$ is zero at any point. Conversely, if $\left[\left.\Omega^{p-1}\right|_{x}\right]$ is constantly zero, then, at any fixed point $x$, there is an $\omega_{x}^{p} \in C^{p+1,1}(\widetilde{\mathfrak{h}}, W)$ with $\partial \omega_{x}^{p}=-\left.\Omega^{p-1}\right|_{x}$. Standard arguments yield that there exists a smooth $\widetilde{\mathfrak{h}}^{p}$-valued 1 -form $\omega^{p}$ on $\mathcal{U}$ such that $\partial \omega^{p}=-\Omega^{p-1}$.

In view of this result, we define essential $(G, \widetilde{G})$-curvature of order $p+1$ the map

$$
\mathcal{R}^{p+1}: \mathcal{U} \rightarrow H^{p, 2}(\widetilde{\mathfrak{h}}, W), \quad \mathcal{R}_{x}^{p+1}:=\left[\left.\Omega^{p-1}\right|_{x}\right] .
$$

It may be considered as a generalization of the $(p+1)^{\text {th }}$-order structure function introduced in [7] for the classical integrability problem.

The following corollary is a direct consequence of Theorem 3.4.

Corollary 3.5 Let $(\widetilde{H} / \widetilde{K}, \widetilde{P})$ be a flat $k$-reductive $\widetilde{G}$-structure for the graded Lie algebra $\widetilde{\mathfrak{h}}$ and $\pi: P \rightarrow M$ a $G$-structure with $G \simeq G_{\sharp} / N_{\sharp}$. Then,

(i) if $P$ is immersible to order $p$ in $\widetilde{P}$ but there exist $x_{o} \in M$ and $r \geq 0$ such that $\left[\left.\widehat{\Omega}_{r}^{p-1}\right|_{x_{o}}\right] \neq$ 0 for any admissible tuple $\left(s, \omega^{0}, \ldots, \omega^{p-1}\right)$, then $P$ is not immersible to order $p+1$;

(ii) if $H^{p, 2}(\widetilde{\mathfrak{h}}, W)=0$ for all $p \geq 0$, then $P$ is locally immersible in $\widetilde{P}$.

\section{Immersions into flat 2-reductive $\widetilde{G}$-structures}

We now restrict to the case of flat 2-reductive $\widetilde{G}$-structures, and $(\widetilde{H} / \widetilde{K}, \widetilde{P})$ always denotes a $\widetilde{G}$-structure of this kind (hence with $\widetilde{\mathfrak{h}}=\widetilde{\mathfrak{h}}^{-1}+\widetilde{\mathfrak{h}}^{0}+\widetilde{\mathfrak{h}}^{1}$ ). We remark that any flat homogeneous $\widetilde{G}$-structure with $\widetilde{G} \subset$ GL( $V)$ irreducible on $V$ and of finite type is either 1-reductive or 2reductive and that, in the second case, $\widetilde{\mathfrak{h}}$ is always determined by a grading of depth 1 of a simple Lie algebra. For the classification of flat 2 -reductive $\widetilde{G}$-structures, see $[10,14]$. 
Definition 4.1 Let $\pi: P \rightarrow M$ be a $G$-structure with $G \simeq G_{\sharp} / N_{\sharp}$. Two admissible tuples $\left(s, \omega^{0}\right)$ and $\left(s^{\prime}, \omega^{\prime 0}\right)$ on the same $\mathcal{U} \subset M$ are strongly equivalent if $s=s^{\prime}$ and $\omega^{\prime 0}=$ $\omega^{0}+\left[\omega^{-1}, \varpi^{1}\right]$ for some $\widetilde{\mathfrak{h}}^{1}$-valued function $\varpi^{1}$.

This definition is motivated by the fact that the essential curvatures of two strongly equivalent tuples are necessarily the same. This can be checked as follows. Let $\left(s, \omega^{0}\right),\left(s, \omega^{\prime 0}\right)$ be strongly equivalent. By definitions, (3.3) and admissibility, one has $\omega^{-1}=\omega^{\prime-1}, \Omega^{-1}=\Omega^{\prime-1}$ and

$$
\Omega^{-1}=-\left[\omega^{-1}, \omega^{0}\right]=-\left[\omega^{-1}, \omega^{\prime 0}\right] .
$$

From this and strong equivalence, it follows that

$$
\begin{aligned}
\Omega^{\prime 0}= & d \omega^{\prime 0}+\frac{1}{2}\left[\omega^{\prime 0}, \omega^{\prime 0}\right] \\
= & \Omega^{0}+d\left[\omega^{-1}, \varpi^{1}\right]+\left[\omega^{0},\left[\omega^{-1}, \varpi^{1}\right]\right]+\frac{1}{2}\left[\left[\omega^{-1}, \varpi^{1}\right],\left[\omega^{-1}, \varpi^{1}\right]\right] \\
= & \Omega^{0}+\left[\Omega^{-1}, \varpi^{1}\right]-\left[\omega^{-1}, d \varpi^{1}\right] \\
& +\left[\omega^{0},\left[\omega^{-1}, \varpi^{1}\right]\right]+\frac{1}{2}\left[\omega^{-1},\left[\varpi^{1},\left[\omega^{-1}, \varpi^{1}\right]\right] \stackrel{(4.1)}{=} \Omega^{0}-\left[\omega^{-1}, \epsilon^{1}\right]\right. \\
\text { with } \epsilon^{1}= & d \varpi^{1}+\left[\omega^{0}, \varpi^{1}\right]+\frac{1}{2}\left[\left[\omega^{-1}, \varpi^{1}\right], \varpi^{1}\right],
\end{aligned}
$$

hence $\left[\Omega^{0}\right]=\left[\Omega^{\prime 0}\right]$.

We also remark that if $H^{1,1}(\widetilde{\mathfrak{h}}, W)=0$, two tuples $\left(s, \omega^{0}\right)$ and $\left(s^{\prime}, \omega^{\prime 0}\right)$ on the same contractible $\mathcal{U}$ are strongly equivalent if and only if $s=s^{\prime}$. Indeed, if $s=s^{\prime}$ Eq. (4.1) holds and $\omega^{\prime 0}-\omega^{0}$ is closed, hence exact.

A similar argument shows that when $H^{2,1}(\widetilde{\mathfrak{h}}, W)=0$ (that is $Z^{2,1}(\widetilde{\mathfrak{h}}, W)=0$ since $\left.\widetilde{\mathfrak{h}}^{2}=0\right)$, two admissible tuples $\left(s, \omega^{0}, \omega^{1}\right)$ and $\left(s^{\prime}, \omega^{\prime 0}, \omega^{\prime 1}\right)$ are equal if and only if $s=s^{\prime}$ and $\omega^{0}=\omega^{\prime 0}$.

In the following, $\pi: P \rightarrow M$ is a $G$-structure with $G \simeq G_{\sharp} / N_{\sharp}, G_{\sharp}, N_{\sharp} \subset \widetilde{G}$ as in (1.1), (1.2). Furthermore, for any admissible tuple $\left(s, \omega^{0}\right)$ of $P$, we denote by $\mathcal{A}_{\left(s, \omega^{0}\right)}$ the set of all admissible tuples $\left(s^{\prime}, \omega^{\prime 0}, \omega^{1}\right)$ with $s^{\prime}=s$ and $\omega^{\prime 0}=\omega^{0}$ and by $\mathcal{R}$ the map which associates to any tuple in $\mathcal{A}_{\left(s, \omega^{0}\right)}$ the corresponding total curvature,

$$
\mathcal{R}: \mathcal{A}_{\left(s, \omega^{0}\right)} \rightarrow \mathcal{C}^{\infty}\left(\mathcal{U}, Z^{2,2}(\widetilde{\mathfrak{h}}, W)\right), \quad \mathcal{R}\left(\left(s, \omega^{0}, \omega^{1}\right)\right):=\Omega^{1} .
$$

The following proposition shows that one can interchange any two strongly equivalent tuples in the analysis of the obstructions to the generalized integrability problem.

Proposition 4.2 Let $\left(s, \omega^{0}\right)$ and $\left(s^{\prime}, \omega^{\prime 0}\right)$ be two strongly equivalent tuples and $\mathcal{R}$ and $\mathcal{R}^{\prime}$ the associated maps defined in (4.3). Then,

$$
\mathcal{R}\left(\mathcal{A}_{\left(s, \omega^{0}\right)}\right)=\mathcal{R}^{\prime}\left(\mathcal{A}_{\left(s^{\prime}, \omega^{\prime 0}\right)}\right) .
$$

Proof Let $\varpi^{1}$ be a 1-form satisfying $\omega^{\prime 0}=\omega^{0}+\left[\omega^{-1}, \varpi^{1}\right]$ and $\epsilon^{1}$ as in (4.2). We observe that $\omega^{1}$ is a solution of $\Omega^{0}=-\left[\omega^{-1}, \omega^{1}\right]$ if and only if $\omega^{\prime 1}=\omega^{1}+\epsilon^{1}$ is a solution of $\Omega^{\prime 0}=-\left[\omega^{\prime-1}, \omega^{\prime 1}\right]$. This shows that the map

$$
\mathcal{T}: \mathcal{A}_{\left(s, \omega^{0}\right)} \rightarrow \mathcal{A}_{\left(s^{\prime}, \omega^{\prime 0}\right)} \quad \mathcal{T}\left(\left(s, \omega^{0}, \omega^{1}\right)\right):=\left(s^{\prime}, \omega^{\prime 0}, \omega^{1}+\epsilon^{1}\right)
$$

is a bijection. Hence, the claim is proved if we can show that the total curvature $\Omega^{\prime 1}$ of $\mathcal{T}\left(s, \omega^{0}, \omega^{1}\right)$ is equal to the total curvature $\Omega^{1}$ of $\left(s, \omega^{0}, \omega^{1}\right)$. By construction, standard 
properties of Lie algebra valued differential forms and the identities $\Omega^{-1}=-\left[\omega^{-1}, \omega^{0}\right]$ and $\Omega^{0}=-\left[\omega^{-1}, \omega^{1}\right]$

$$
\begin{aligned}
\Omega^{\prime 1}= & d \omega^{1}+\left[\omega^{\prime 0}, \omega^{\prime 1}\right] \\
= & \Omega^{1}+d \epsilon^{1}+\left[\omega^{0}, \epsilon^{1}\right]+\left[\left[\omega^{-1}, \varpi^{1}\right], \omega^{1}\right]+\left[\left[\omega^{-1}, \varpi^{1}\right], \epsilon^{1}\right] \\
= & \Omega^{1}+d\left[\omega^{0}, \varpi^{1}\right]+\frac{1}{2} d\left[\left[\omega^{-1}, \varpi^{1}\right], \varpi^{1}\right]+\left[\omega^{0}, d \varpi^{1}\right]+\left[\omega^{0},\left[\omega^{0}, \varpi^{1}\right]\right] \\
& +\frac{1}{2}\left[\omega^{0},\left[\left[\omega^{-1}, \varpi^{1}\right], \varpi^{1}\right]\right]+\left[\left[\omega^{-1}, \varpi^{1}\right], \omega^{1}\right]+\left[\left[\omega^{-1}, \varpi^{1}\right], d \varpi^{1}\right] \\
& +\left[\left[\omega^{-1}, \varpi^{1}\right],\left[\omega^{0}, \varpi^{1}\right]\right]+\frac{1}{2}\left[\left[\omega^{-1}, \varpi^{1}\right],\left[\left[\omega^{-1}, \varpi^{1}\right], \varpi^{1}\right]\right] \\
= & \Omega^{1}+\frac{1}{2}\left[\left[\Omega^{-1}, \varpi^{1}\right], \varpi^{1}\right]+\frac{1}{2}\left[\left[\left[\omega^{-1}, \omega^{0}\right], \varpi^{1}\right], \varpi^{1}\right]+\left[\Omega^{0}, \varpi^{1}\right] \\
& +\left[\left[\omega^{-1}, \omega^{1}\right], \varpi^{1}\right]-\frac{1}{2}\left[\omega^{-1},\left[\left[\omega^{0}, \varpi^{1}\right], \varpi^{1}\right]\right]-\left[\omega^{-1},\left[\omega^{1}, \varpi^{1}\right]\right] \\
& -\frac{1}{2}\left[\omega^{-1},\left[d \varpi^{1}, \varpi^{1}\right]\right]-\frac{1}{6}\left[\omega^{-1},\left[\left[\left[\omega^{-1}, \varpi^{1}\right], \varpi^{1}\right], \varpi^{1}\right]\right] \\
= & \Omega^{1}-\frac{1}{2}\left[\omega^{-1},\left[\left[\omega^{0}, \varpi^{1}\right], \varpi^{1}\right]\right]-\left[\omega^{-1},\left[\omega^{1}, \varpi^{1}\right]\right] \\
& -\frac{1}{2}\left[\omega^{-1},\left[d \varpi^{1}, \varpi^{1}\right]\right]-\frac{1}{6}\left[\omega^{-1},\left[\left[\left[\omega^{-1}, \varpi^{1}\right], \varpi^{1}\right], \varpi^{1}\right]\right] .
\end{aligned}
$$

Since $\widetilde{\mathfrak{h}}^{2}=0$, all terms except $\Omega^{1}$ are trivial; hence, $\Omega^{1}=\Omega^{\prime 1}$.

Theorem 4.3 Let $H^{1,1}(\widetilde{\mathfrak{h}}, W)=H^{2,1}(\widetilde{\mathfrak{h}}, W)=0$. Given $0 \leq p \leq 2$, if $P$ is locally immersible into $\widetilde{P}$ to order $p$, then:

(i) for any $x \in M$, the value $\left[\left.\Omega^{p-1}\right|_{x}\right]$ of the essential $(G, \widetilde{G})$-curvature of an admissible tuple $\left(s, \omega^{0}, \ldots, \omega^{p-1}\right)$ depends only on the frame $u=\left.s_{x} \in P\right|_{x}$; this determines a well-defined map

$$
\mathcal{R}^{p+1}: P \rightarrow H^{p, 2}(\widetilde{\mathfrak{h}}, W) \quad \mathcal{R}^{p+1}(u):=\left[\left.\Omega^{p-1}\right|_{x}\right] ;
$$

(ii) the map (4.4) is $G_{\sharp}$-equivariant with respect to its action on $P$ via the isomorphism $G \simeq G_{\sharp} / N_{\sharp}$ and its standard actions on $W$ and $\widetilde{\mathfrak{h}}$;

(iii) the map (4.4) vanishes identically if and only if $P$ is locally immersible into $\widetilde{P}$ to order $p+1$.

Proof Let $s, s^{\prime}: \mathcal{U} \subset M \rightarrow P$ be two fixed local sections on the same contractible $\mathcal{U}$, hence of the form $s^{\prime}=s \circ g^{-1}$ for some $g: \mathcal{U} \rightarrow G$. If $p_{\sharp}: G_{\sharp} \rightarrow G=G_{\sharp} / N_{\sharp}$ is the natural projection, then $g=p_{\sharp} \circ g_{\sharp}$ for some $g_{\sharp}: \mathcal{U} \rightarrow G_{\sharp}$.

As usual, for any admissible tuple $\left(s, \omega^{0}, \ldots, \omega^{p-1}\right)$ associated with $s$, we use the frame $s_{x}$ to identify at each $x \in \mathcal{U}$ the 1-forms $\left.\omega^{r}\right|_{x}$ and the associated curvatures $\Omega^{r}$ with elements in $C(\widetilde{\mathfrak{h}}, W)$. On the other hand, for the admissible tuples $\left(s^{\prime}, \omega^{\prime 0}, \ldots, \omega^{\prime p-1}\right)$ associated with $s^{\prime}$, we use $s_{x}^{\prime}$ (and not $s_{x}$ ) to make the corresponding identifications for $\left.\omega^{\prime r}\right|_{x}$ and $\Omega^{\prime r}$.

Case $p=0$. Let $\omega^{-1}, \omega^{-1}$ be the $W$-valued 1 -forms associated with $s, s^{\prime}$ as in (3.3) and $\Omega^{-1}, \Omega^{\prime-1}$ the corresponding total $(G, \widetilde{G})$-curvatures. A direct computation shows that

$$
\Omega^{\prime-1}=g_{\sharp} \cdot \Omega^{-1}+\partial\left(R_{g_{\sharp}^{-1}}\left(d g_{\sharp} \circ s^{\prime}\right)\right),
$$


where we denote by $R_{g_{\sharp}}$ the natural right action of $g_{\sharp}$ on $T G_{\sharp}$. It follows that $\left[\Omega^{\prime-1}\right]=$ $g_{\sharp} \cdot\left[\Omega^{-1}\right]$. From this, the transitivity of the action of $G$ on the fibers of $P$, and the fact that $s_{x}^{\prime}=s_{x}$ implies $\left.g_{\sharp}\right|_{x}=e$, (i) and (ii) follow. Claim (iii) follows from Theorem 3.4.

Case $p=1$. Let $P$ be immersible in $\widetilde{P}$ to order 1 . Consider an admissible tuple $\left(s, \omega^{0}\right)$ with associated total $(G, \widetilde{G})$-curvature $\Omega^{0}$. By definition, it satisfies $\Omega^{-1}=-\partial \omega^{0}$. Since $H^{1,1}(\widetilde{\mathfrak{h}}, W)=0$, we know that two admissible tuples $\left(s, \omega^{0}\right)$ and $\left(\widehat{s}, \widehat{\omega}^{0}\right)$ with $s=\widehat{s}$ are always strongly equivalent, hence with the same essential curvature of order 2 (see remarks before Proposition 4.2).

Now, Eq. (4.5) and the fact that the generalized Spencer operator is $G_{\sharp}$-equivariant yield

$$
\Omega^{\prime-1}=g_{\sharp} \cdot\left(-\partial \omega^{0}\right)+\partial\left(R_{g_{\sharp}^{-1}}\left(d g_{\sharp} \circ s^{\prime}\right)\right)=-\partial\left(g_{\sharp} \cdot \omega^{0}-R_{g_{\sharp}^{-1}}\left(d g_{\sharp} \circ s^{\prime}\right)\right),
$$

that is $\left(s^{\prime}, g_{\sharp} \cdot\left(\omega^{0} \circ s^{-1}\right)-R_{g_{\sharp}^{-1}}\left(d g_{\sharp}\right)\right)$ is admissible. Further, a direct computation shows that the total curvature $\Omega^{\prime 0}$ of this admissible tuple is $\Omega^{\prime 0}=g_{\sharp} \cdot \Omega^{0}$. By the above remark, this implies that $\left[\Omega^{\prime 0}\right]=g_{\sharp} \cdot\left[\Omega^{0}\right]$ independently of the choice of $\omega^{0}$ and $\omega^{\prime 0}$. This yields (i), (ii), and (iii) with the same arguments of the case $p=0$.

Case $p=2$. Let $P$ be immersible in $\widetilde{P}$ to order 2 . Since $H^{1,1}(\widetilde{\mathfrak{h}}, W)=H^{2,1}(\widetilde{\mathfrak{h}}, W)=0$, by the remarks before Proposition 4.2 any two admissible tuples $\left(s, \omega^{0}\right),\left(\widehat{s}, \widehat{\omega}^{0}\right)$ with $s=\widehat{s}$ are strongly equivalent, and the sets $\mathcal{A}_{\left(s, \omega^{0}\right)}, \mathcal{A}_{\left.\widehat{s}, \widehat{\omega}^{0}\right)}$ consist both of just one element, say $\omega^{1}$ and $\widehat{\omega}^{1}$, respectively. By Proposition 4.2, the total $(G, \widetilde{G})$-curvatures of $\left(s, \omega^{0}, \omega^{1}\right)$ and $\left(\widehat{s}, \widehat{\omega}^{0}, \widehat{\omega}^{1}\right)$ coincide, meaning that such curvature depends only on $s$.

Let us consider now an admissible tuple $\left(s, \omega^{0}, \omega^{1}\right)$ and the admissible tuple $\left(s^{\prime}, \omega^{\prime 0}, \omega^{\prime 1}\right)$ where $\omega^{\prime 0}$ is as in the previous case and $\omega^{\prime 1}=g_{\sharp} \cdot\left(\omega^{1} \circ S^{-1}\right)$. The associated curvatures satisfy $\Omega^{\prime 1}=g_{\sharp} \cdot \Omega^{1}$. By the above remark, this implies that $\Omega^{\prime 1}=g_{\sharp} \cdot \Omega^{1}$ independently of the choice of $\left(\omega^{0}, \omega^{1}\right)$ and $\left(\omega^{\prime 0}, \omega^{1}\right)$. This yields (i), (ii), and (iii) with the same arguments as above.

\section{Applications}

\subsection{Riemannian immersions into spaces of constant curvature}

Let $(\widetilde{M}, \widetilde{g})$ be a Riemannian manifold of dimension $\widetilde{n}$ and $\widetilde{\pi}: \widetilde{P}=O_{\widetilde{g}}(\widetilde{M}) \rightarrow \widetilde{M}$ the $\mathrm{O}_{\widetilde{n}}(\mathbb{R})$-structure given by the orthonormal frames

$$
O_{\widetilde{g}}(\widetilde{M})=\left\{\left(e_{i}\right) \in L(\widetilde{M}) \mid \widetilde{g}\left(e_{i}, e_{j}\right)=\delta_{i j}, \quad 1 \leq i, j \leq \widetilde{n}\right\}
$$

Any $n$-dimensional submanifold $M \subset \widetilde{M}$ is $\widetilde{P}$-regular so that one can always consider the induced $G$-structure $\pi: P \rightarrow M$, which is the orthonormal frame bundle of the Riemannian metric $g$ induced on $M$ by $\widetilde{g}$. It follows that, given two Riemannian manifolds $(M, g)$ and $(\widetilde{M}, \widetilde{g})$, the local immersibility of $\pi: O_{g}(M) \rightarrow M$ into $\widetilde{\pi}: O_{\widetilde{g}}(\widetilde{M}) \rightarrow \widetilde{M}$ is equivalent to the existence of local isometric immersions of $(M, g)$ into $(\widetilde{M}, \widetilde{g})$.

Let us consider now the problem of the existence of a local isometric immersion of an $n$-dimensional Riemannian manifold $(M, g)$ into a space of constant curvature $k_{o}$ that is of the local immersibility of $P=O_{g}(M)$ in the homogeneous $\widetilde{G}$-structure $(\widetilde{H} / \widetilde{K}, \widetilde{P})$ with 


$$
\widetilde{H}=\left\{\begin{array}{ll}
\operatorname{SO}_{\widetilde{n}}(\mathbb{R}) \ltimes \mathbb{R}^{\tilde{n}} & \text { if } k_{o}=0 \\
\operatorname{SO}_{\widetilde{n}+1}(\mathbb{R}) & \text { if } k_{o}=1 \\
\operatorname{SO}_{\tilde{n}, 1}(\mathbb{R}) & \text { if } k_{o}=-1
\end{array} \quad, \quad \widetilde{K}=\operatorname{SO}_{\widetilde{n}}(\mathbb{R}),\right.
$$

and $\widetilde{P}$ the orthonormal frame bundle of the homogeneous Riemannian manifold $(\tilde{M}=$ $\widetilde{H} / \widetilde{K}, \widetilde{g})$, that is, $\mathbb{R}^{\widetilde{n}}, S^{\widetilde{n}}$ or $H^{\widetilde{n}}$ with their standard metrics of curvatures $k_{o}=0,1,-1$ respectively.

These homogeneous spaces are reductive with quasi-graded Lie algebras

$$
\widetilde{\mathfrak{h}}=\widetilde{\mathfrak{h}}^{-1}+\widetilde{\mathfrak{h}}^{0} \quad \text { where } \quad \widetilde{\mathfrak{h}}^{-1}=\mathbb{R}^{\widetilde{n}}, \quad \widetilde{\mathfrak{h}}^{0}=\mathfrak{s o}_{\widetilde{n}}(\mathbb{R}),
$$

with Lie brackets defined by

$$
[A, v]=A v \in \widetilde{\mathfrak{h}}^{-1}, \quad\left[v_{1}, v_{2}\right]=k_{o}\left(v_{2} \otimes\left\langle v_{1}, \cdot\right\rangle-v_{1} \otimes\left\langle v_{2}, \cdot\right\rangle\right) \in \widetilde{\mathfrak{h}}^{0},
$$

for any $v, v_{1}, v_{2} \in \widetilde{\mathfrak{h}}^{-1}$ and $A \in \widetilde{\mathfrak{h}}^{0}$.

For our purposes, it is convenient to consider the decomposition of $\widetilde{\mathfrak{h}}^{0}$ into the following vector subspaces

$$
\begin{aligned}
\mathfrak{s o}_{\tilde{n}}(\mathbb{R}) & =\left\{\left(\begin{array}{cc}
A & -B^{t} \\
B & D
\end{array}\right) \mid A \in \mathfrak{s o}_{n}(\mathbb{R}), B \in \mathbb{R}^{\widetilde{n}-n} \otimes\left(\mathbb{R}^{n}\right)^{*}, D \in \mathfrak{s o}_{\tilde{n}-n}(\mathbb{R})\right\} \\
& =\mathfrak{s o}(W)+W^{\perp} \otimes W^{*}+\mathfrak{s o}\left(W^{\perp}\right) \text { with } W=\mathbb{R}^{n}, W^{\perp}=\mathbb{R}^{\widetilde{n}-n} .
\end{aligned}
$$

In $\widetilde{\mathfrak{h}}^{0}$, there is only one annihilator, that is, $\mathfrak{c}_{1}^{0}=\mathfrak{s o}\left(W^{\perp}\right)$, and from now on, we consider $\mathfrak{c}_{1}^{0 \perp}=\mathfrak{s o}(W)+W^{\perp} \otimes W^{*}$ as a fixed complementary subspace.

By the results of Sect. 3.2, there exists a local isometric immersion of $(M, g)$ in $(\widetilde{M}, \widetilde{g})$ if and only if there is a pair $\left(s, \omega^{0}\right)$ consisting of:

1. a local section $s: \mathcal{U} \subset M \rightarrow O_{g}(M)$, i.e., a field of $g$-orthonormal frames which we use to identify any tangent space $T_{x} M$ with $W=\mathbb{R}^{n}$ and to define the 1-form

$$
\left.\omega^{-1}\right|_{x}(w)=\left(w^{1}, \ldots, w^{n}, 0, \ldots, 0\right), \quad w=\left(w^{i}\right) \in W \simeq T_{x} M, \quad x \in \mathcal{U} ;
$$

2. a $\mathfrak{s o}_{\tilde{n}}(\mathbb{R})$-valued local 1 -form $\omega^{0}$ satisfying the equations

$$
\begin{aligned}
& {\left[\omega^{-1}, \omega^{0}\right]=-d \omega^{-1},} \\
& \Omega^{0}=d \omega^{0}+\frac{1}{2}\left[\omega^{0}, \omega^{0}\right]=0 .
\end{aligned}
$$

According to (5.1), we decompose $\omega^{0}=\omega^{\mathfrak{s o}(W)}+\omega^{W^{\perp} \otimes W^{*}}+\omega^{\mathfrak{s o}\left(W^{\perp}\right)}$ so that (5.2) is equivalent to the following equations for any $x \in \mathcal{U}$ and $w_{1}, w_{2} \in W$

$$
\begin{aligned}
& -\omega_{x}^{\mathfrak{s o}(W)}\left(w_{2}\right)\left(w_{1}\right)+\omega_{x}^{\mathfrak{s o}(W)}\left(w_{1}\right)\left(w_{2}\right)=-\left.d \omega^{-1}\right|_{x}\left(w_{1}, w_{2}\right), \\
& -\omega_{x}^{W^{\perp} \otimes W^{*}}\left(w_{2}\right)\left(w_{1}\right)+\omega_{x}^{W^{\perp} \otimes W^{*}}\left(w_{1}\right)\left(w_{2}\right)=0 .
\end{aligned}
$$

By classical arguments, Eq. (5.4) has a unique solution $\omega^{\mathfrak{s o}(W)}$, namely the connection 1form of the Levi-Civita covariant derivative $\nabla^{L C}$ of $(M, g)$. On the other hand, if $N M$ is the trivial vector bundle $\mathrm{NM}=\mathcal{U} \times W^{\perp} \rightarrow \mathcal{U}$, Eq. (5.5) means that $\omega^{W^{\perp} \otimes W^{*}}$ can be identified with a symmetric local section $\Pi$ of $\otimes^{2} T M^{*} \otimes N M$. We remark that, by the identification $W^{\perp}=\mathbb{R}^{\widetilde{n}-n}$, the trivial bundle $N M$ has a natural fiber metric, which we denote by $g^{\perp}$, and $\omega^{\mathfrak{s o}\left(W^{\perp}\right)}$ is the connection 1-form of a covariant derivative $\nabla^{\perp}$ on $N M$ compatible with $g^{\perp}$.

Using again (5.1), we now decompose $\Omega^{0}$ into

$$
\Omega^{0}=\Omega^{\mathfrak{s o}(W)}+\Omega^{W^{\perp} \otimes W^{*}}+\Omega^{\mathfrak{s o}\left(W^{\perp}\right)},
$$


and we observe that the curvature $\widehat{\Omega}_{1}^{0}$ of level 1 defined in (3.5) and the complementary curvature $\widetilde{\Omega}_{0}^{0}$ of level 0 defined in (3.7) are equal to

$$
\widehat{\Omega}_{1}^{0}=\Omega^{\mathfrak{s o}(W)}+\Omega^{W^{\perp} \otimes W^{*}} \quad \text { and } \quad \widetilde{\Omega}_{0}^{0}=\Omega^{\mathfrak{s o}\left(W^{\perp}\right)} .
$$

Recall that $\Omega^{0}=\widehat{\Omega}_{1}^{0}+\widetilde{\Omega}_{0}^{0}$. A simple computation shows

$$
\begin{aligned}
\Omega^{\mathfrak{s o}(W)}= & d \omega^{\mathfrak{s o}(W)}+\frac{1}{2}\left[\omega^{\mathfrak{s o}(W)}, \omega^{\mathfrak{s o}(W)}\right]-\left(\omega^{W^{\perp} \otimes W^{*}}\right)^{t} \cdot \omega^{W^{\perp} \otimes W^{*}} \\
& +\frac{1}{2}\left[\omega^{-1}, \omega^{-1}\right], \\
\Omega^{W^{\perp} \otimes W^{*}}= & d \omega^{W^{\perp} \otimes W^{*}}+\omega^{W^{\perp} \otimes W^{*}} \cdot \omega^{\mathfrak{s o}(W)}+\omega^{\mathfrak{s o}\left(W^{\perp}\right)} \cdot \omega^{W^{\perp} \otimes W^{*}}, \\
\Omega^{\mathfrak{s o}\left(W^{\perp}\right)}= & d \omega^{\mathfrak{s o}\left(W^{\perp}\right)}+\frac{1}{2}\left[\omega^{\mathfrak{s o}\left(W^{\perp}\right)}, \omega^{\mathfrak{s o}\left(W^{\perp}\right)}\right]-\omega^{W^{\perp} \otimes W^{*}} \cdot\left(\omega^{W^{\perp} \otimes W^{*}}\right)^{t} .
\end{aligned}
$$

Let $\nabla=\nabla^{L C}+\nabla^{\perp}$ be the covariant derivative on $T M \oplus N M$ determined by the connection 1-form $\omega^{\mathfrak{s o}(W)}+\omega^{\mathfrak{s o}\left(W^{\perp}\right)}$ and denote by $R$ its curvature. We remark that $\nabla$ is compatible with the fiber metric $g+g^{\perp}$.

Equation (5.3) splits into the equations $\widehat{\Omega}_{1}^{0}=\Omega^{\mathfrak{s o}(W)}+\Omega^{W^{\perp} \otimes W^{*}}=0$ and $\widehat{\Omega}_{0}^{0}=$ $\Omega^{\mathfrak{s o}\left(W^{\perp}\right)}=0$. One can check that the first corresponds to the Gauss-Codazzi equations

$$
\begin{aligned}
& g\left(R_{X Y} Z, W\right)-g^{\perp}(\Pi(Y, Z), \Pi(X, W))+g^{\perp}(\Pi(X, Z), \Pi(Y, W)) \\
& \quad+k_{o}(g(X, Z) g(Y, W)-g(Y, Z) g(X, W))=0,
\end{aligned}
$$

and

$$
\left(\nabla_{X} \Pi\right)(Y, Z)-\left(\nabla_{Y} \Pi\right)(X, Z)=0,
$$

for any $X, Y, Z, W \in \mathfrak{X}(M)$. The second one corresponds to the Ricci equations

$$
\begin{gathered}
g^{\perp}\left(R_{X Y} \mu, v\right)+\sum_{i=1}^{n} g^{\perp}\left(\Pi\left(X, e_{i}\right), \mu\right) g^{\perp}\left(\Pi\left(Y, e_{i}\right), v\right) \\
-\sum_{i=1}^{n} g^{\perp}\left(\Pi\left(X, e_{i}\right), v\right) g^{\perp}\left(\Pi\left(Y, e_{i}\right), \mu\right)=0,
\end{gathered}
$$

for any $X, Y \in \mathfrak{X}(M)$ and local sections $\mu, v$ of $N M$, with $\left\{e_{i}\right\}$ a fixed local orthonormal frame field of $(M, g)$.

By these observations, it follows that, in this case, the results of Sect. 3.2 yield the classical result that a Riemannian manifold $(M, g)$ has a local isometric immersion in a space of constant curvature $k_{o}$ if and only if there exists a metric bundle $\left(N M, g^{\perp}\right)$, a compatible connection $\nabla^{\perp}$, and a symmetric tensor $\Pi$ that satisfy the Gauss-Codazzi-Ricci equations (see, e.g., [21]).

Remark 5.1 For Riemannian immersions into Euclidean spaces, i.e., with $k_{o}=0$, the homogeneous $\widetilde{G}$-structure $(\widetilde{H} / \widetilde{K}, \widetilde{P})$ is flat $k$-reductive, and one can discuss the obstructions to the generalized integrability problem as in Sect. 3.3. In this situation, the only relevant cohomology groups are $H^{0,2}(\widetilde{\mathfrak{g}}, W)$ and $H^{1,2}(\widetilde{\mathfrak{g}}, W)$ with $\widetilde{\mathfrak{g}}=\mathfrak{s o}_{\tilde{n}}(\mathbb{R})$ and $W=\mathbb{R}^{n}$. One can directly check that $H^{0,2}\left(\mathfrak{s o}_{\tilde{n}}(\mathbb{R}), \mathbb{R}^{n}\right)=0$ (this is equivalent to the fact that (5.4) and (5.5) are always solvable) and

$$
H^{1,2}\left(\mathfrak{s o}_{\tilde{n}}(\mathbb{R}), W\right) \simeq H^{1,2}\left(\mathfrak{s o}_{n}(\mathbb{R}), W\right)+W^{\perp} \otimes \mathrm{R}^{2,1}+\mathfrak{s o}\left(W^{\perp}\right) \otimes \Lambda^{2} W^{*},
$$


where $\mathrm{R}^{2,1}$ is the unique $\mathfrak{g l}(W)$-irreducible submodule of $W^{*} \otimes \Lambda^{2} W^{*}$ which is different from $\Lambda^{3} W^{*}$ (that is the kernel of complete antysimmetrization).

As $\widetilde{\mathfrak{h}}^{1}=0$, the cohomology class of $\Omega^{0}$ is trivial if and only if each of the three components

$$
\begin{aligned}
& \left.\Omega^{\mathfrak{s o}(W)}\right|_{x} \in H^{1,2}\left(\mathfrak{s o}_{n}(\mathbb{R}), W\right),\left.\quad \Omega^{W^{\perp} \otimes W^{*}}\right|_{x} \in W^{\perp} \otimes \mathrm{R}^{2,1} \quad \text { and } \\
& \left.\Omega^{\mathfrak{s o}(W)}\right|_{x} \in \mathfrak{s o}\left(W^{\perp}\right) \otimes \Lambda^{2} W^{*},
\end{aligned}
$$

is equal to zero, that is, the Gauss, the Codazzi, and the Ricci equations are, respectively, satisfied.

Remark 5.2 We observe that the cohomology groups introduced in Sect. 3.3 are different from those considered by Rosly and Schwarz in [16]. In that paper, the authors claimed that the only obstructions to the generalized integrability problem into flat $k$-reductive $\widetilde{G}$-structures are represented by the non-trivial classes in such cohomology groups ([16, Appendix C]). On the other hand, in the case of Riemannian immersions into an Euclidean space, one can check that the only non-trivial Rosly and Schwarz cohomology group is identifiable with the subspace $H^{1,2}\left(\mathfrak{s o}_{n}(\mathbb{R}), \mathbb{R}^{n}\right)$ of $H^{1,2}\left(\mathfrak{s o}_{\tilde{n}}(\mathbb{R}), \mathbb{R}^{n}\right)$. This means that the set of Rosly and Schwarz obstructions corresponds just to the Gauss equation and seems therefore not to be complete.

\subsection{Conformal immersions into a conformally flat space}

Let $(\tilde{M}, \widetilde{g})$ be an $\tilde{n}$-dimensional Riemannian manifold and $\tilde{\pi}: \widetilde{P}=\operatorname{CO}_{\widetilde{g}}(\widetilde{M}) \rightarrow \widetilde{M}$ the $\mathrm{CO}_{\widetilde{n}}(\mathbb{R})$-structure of conformal frames

$$
\mathrm{CO}_{\widetilde{g}}(\tilde{M})=\left\{\left(e_{i}\right) \in L(\tilde{M}) \mid \widetilde{g}\left(e_{i}, e_{j}\right)=c \delta_{i j}, 1 \leq i, j \leq \widetilde{n}, c \in(0,+\infty)\right\} .
$$

Any $n$-dimensional submanifold $M \subset \widetilde{M}$ is $\widetilde{P}$-regular and has an induced $G$-structure $\pi: P \rightarrow M$, naturally identifiable with the bundle of conformal frames of the induced Riemannian metric $g$. It follows that, given two Riemannian manifolds $(M, g),(\widetilde{M}, \widetilde{g})$, the existence of local conformal immersions of $(M, g)$ into $(\widetilde{M}, \widetilde{g})$ is equivalent to the existence of a local immersion of $\pi: \mathrm{CO}_{g}(M) \rightarrow M$ into $\widetilde{\pi}: \mathrm{CO}_{\widetilde{g}}(\widetilde{M}) \rightarrow \widetilde{M}$. Due to this, the existence of a local conformal immersion of $(M, g)$ in the homogeneous conformally flat space $\left(S^{\tilde{n}}, \widetilde{g}\right)$ is equivalent to the local immersibility of $\pi: \mathrm{CO}_{g}(M) \rightarrow M$ in the homogeneous $\widetilde{G}$-structure $\left(S^{\widetilde{n}}=\mathrm{SO}_{\widetilde{n}+1,1}(\mathbb{R}) / \widetilde{K}, \widetilde{P}\right)$ with $\widetilde{P}=\operatorname{CO}_{\widetilde{g}}\left(S^{\widetilde{n}}\right)$ and $\widetilde{K}$ an appropriate parabolic subgroup.

This homogeneous $\widetilde{G}$-structure is flat $k$-reductive and associated with the graded Lie algebra $\widetilde{\mathfrak{h}}=\mathfrak{s o} \widetilde{n}+1,1(\mathbb{R})$ with grading

$$
\widetilde{\mathfrak{h}}=\widetilde{\mathfrak{h}}^{-1}+\widetilde{\mathfrak{h}}^{0}+\widetilde{\mathfrak{h}}^{1}=V+\mathfrak{c o}(V)+V^{*}, \text { where } \quad V=\mathbb{R}^{\widetilde{n}},
$$

and Lie brackets determined by the standard action of $\operatorname{co}(V)$ on $V$ and $V^{*}$ and the bracket between $\alpha \in V^{*}$ and $v \in V$ defined by

$$
[\alpha, v]=v \otimes \alpha-\natural(\alpha \otimes v)+\alpha(v) I .
$$

Here, $\downarrow: V^{*} \otimes V \rightarrow V \otimes V^{*}$ is the natural isomorphism induced by the standard scalar product $\langle\cdot, \cdot\rangle$ of $V=\mathbb{R}^{\widetilde{n}}$. The isotropy subalgebra is

$$
\widetilde{\mathfrak{k}}=\widetilde{\mathfrak{h}}^{0}+\widetilde{\mathfrak{h}}^{1}=\mathfrak{c o}(V)+V^{*} .
$$

We recall that when $\widetilde{n} \geq 3, \widetilde{\mathfrak{h}}$ is the maximal prolongation of $\mathfrak{c o}(V)([9,20])$.

For the case of conformal immersions into a conformally flat Riemannian space, the analogues of the Gauss-Codazzi-Ricci equations are provided by the following theorem. 
We first need to fix some notation. Let $(M, g)$ be an $n$-dimensional Riemannian manifold with Levi-Civita connection $\nabla^{L C}$ and $\widetilde{n} \geq n$. Given two symmetric tensors $b, b^{\prime}$ of type $(0,2)$ on $M$, we also denote by $b \otimes b^{\prime}$ their Kulkarni-Nomizu product, namely the $(0,4)$-tensor defined by

$$
\begin{aligned}
b \oplus b^{\prime}(X, Y, Z, W)= & b(X, Z) b^{\prime}(Y, W)+b(Y, W) b^{\prime}(X, Z) \\
& -b(X, W) b^{\prime}(Y, Z)-b(Y, Z) b^{\prime}(X, W) .
\end{aligned}
$$

Theorem 5.3 Given a conformal immersion $\iota:(M, g) \longrightarrow\left(\mathbb{R}^{\tilde{n}},\langle\cdot, \cdot\rangle\right)$ with $\iota^{*}\langle\cdot, \cdot\rangle=e^{2 f} g$ for a smooth function $f$, the quadruple $\left(\nabla^{\perp}, \Pi, B, D\right)$ formed by the restriction $\nabla^{\perp}$ of the Levi-Civita connection of $\left(\mathbb{R}^{\tilde{n}},\langle\cdot, \cdot\rangle\right)$ along the normal bundle $N M$ of $M$ and the tensors

$$
\begin{aligned}
& \Pi=e^{-f} \cdot \bar{\Pi}, \\
& B=\operatorname{Hess}(f)-d f \odot d f+\frac{1}{2} g(\nabla f, \nabla f) g, \\
& D=e^{f} \cdot \bar{\Pi}(\nabla f, \cdot),
\end{aligned}
$$

where $\bar{\Pi}$ is the second fundamental form of $\left(M, e^{2 f} g\right) \subset\left(\mathbb{R}^{\tilde{n}},\langle\cdot, \cdot\rangle\right)$ and $\nabla f$ and $\operatorname{Hess}(f)$ are the gradient and the Hessian of $f$ w.r.t. $g$, satisfies the following equations for any $X, Y, Z, W \in \mathfrak{X}(M)$ and $\mu, v \in \Gamma(N M)$ :

$$
\begin{gathered}
g\left(R_{X Y} Z, W\right)-\langle\Pi(Y, Z), \Pi(X, W)\rangle+\langle\Pi(X, Z), \Pi(Y, W)\rangle \\
=-g \oplus B(X, Y, Z, W), \\
\left(\nabla_{X} \Pi\right)(Y, Z)-\left(\nabla_{Y} \Pi\right)(X, Z)=D(X) g(Y, Z)-D(Y) g(X, Z), \\
\left\langle R_{X Y} \mu, v\right\rangle+\sum_{i=1}^{n}\left\langle\Pi\left(X, e_{i}\right), \mu\right\rangle\left\langle\Pi\left(Y, e_{i}\right), v\right\rangle \\
-\sum_{i=1}^{n}\left\langle\Pi\left(X, e_{i}\right), v\right\rangle\left\langle\Pi\left(Y, e_{i}\right), \mu\right\rangle=0, \\
\left(\nabla_{X} B\right)(Y, Z)+\langle D(X), \Pi(Y, Z)\rangle \text { is symmetric in } X, Y, \\
\left(\nabla_{X} D_{b}\right)(Y, \mu)+\sum_{i=1}^{n}\left\langle\Pi\left(X, E_{i}\right), \mu\right\rangle B\left(Y, E_{i}\right) \text { is symmetric in } X, Y,
\end{gathered}
$$

where $R$ is the curvature of the covariant derivative $\nabla=\nabla^{L C}+\nabla^{\perp}$ on the vector bundle $T M \oplus N M \rightarrow M,\left\{e_{i}\right\}$ a fixed local orthonormal frame field of $(M, g)$ and $b: N M \rightarrow N M^{*}$ the natural isomorphism induced by $\left.\langle\cdot, \cdot\rangle\right|_{N M}$.

Proof The Eqs. (5.7)-(5.9) are a consequence of the Gauss-Codazzi-Ricci equations for the isometric immersion $\left(M, e^{2 f} g\right) \subset\left(\mathbb{R}^{\widetilde{n}},\langle\cdot, \cdot\rangle\right)$ and the explicit expressions of Levi-Civita connection and curvature of $g^{\prime}=e^{2 f} g$ in terms of $g$ and $f$ (see, e.g., [6, Thm.1.159]; caution: We use a definition of curvature which is opposite in sign to the one in [6]). Equations (5.10) and (5.11) follow from the definitions of $B$ and $D$ by a somehow long but straightforward computation.

The contents of Sect. 3.3 yield the following theorem which shows that the above equations are actually necessary and sufficient conditions for the existence of local conformal immersions into conformally flat spaces. This is a result that was actually first obtained by Akivis ([1], see also [2, Theorem 3.1.5]) using the method of moving frames. 
In the statement of this theorem, given an open subset $\mathcal{U} \subset M$, we denote by $N M=$ $\mathcal{U} \times W^{\perp} \rightarrow \mathcal{U}$ the trivial vector bundle with fiber $W^{\perp}=\mathbb{R}^{\widetilde{n}-n}$ and natural fiber metric determined by the standard inner product $\left.\langle\cdot, \cdot\rangle\right|_{W^{\perp}}$.

Theorem 5.4 Let $(M, g)$ be an $n$-dimensional Riemannian manifold and $\widetilde{n} \geq n, \tilde{n} \geq 3$. Then, for any $x \in M$, there exists a local conformal immersion of $(M, g)$ around $x$ into $\left(S^{\tilde{n}}, \widetilde{g}\right)$ if and only if for some neighborhood $\mathcal{U} \subset M$ of $x$, there is a quadruple $\left(\nabla^{\perp}, \Pi, B, D\right)$ consisting of

(i) a metric connection $\nabla^{\perp}:\left.T M\right|_{\mathcal{U}} \times N M \rightarrow N M$,

(ii) a local section $\Pi$ of $S^{2} T^{*} M \otimes N M$,

(iii) a local section $B$ of $S^{2} T^{*} M$,

(iv) a local section $D$ of $T^{*} M \otimes N M$,

which satisfies (5.7)-(5.11).

Proof According to the decomposition (5.1) of $\mathfrak{s o}(V)$, we consider the decomposition of $\widetilde{\mathfrak{h}}^{0}=\mathfrak{c o}(V)$ into the vector subspaces

$$
\mathfrak{c o}(V)=\mathfrak{s o}(V)+\mathbb{R}=\mathfrak{s o}(W)+W^{\perp} \otimes W^{*}+\mathfrak{s o}\left(W^{\perp}\right)+\mathbb{R} .
$$

We also consider the decomposition of $\widetilde{\mathfrak{h}}^{1}=V^{*}$ given by

$$
V^{*}=W^{*}+\left(W^{\perp}\right)^{*} .
$$

Since $\widetilde{\mathfrak{h}}^{1} \neq(0)$, by the results of Sect. 3.2, there exists a local conformal immersion of $(M, g)$ into $\left(S^{\widetilde{n}}, \widetilde{g}\right)$ if and only if there is an admissible triple $\left(s, \omega^{0}, \omega^{1}\right)$, that is, a triple consisting of:

1. a local section $s: \mathcal{U} \subset M \rightarrow \mathrm{CO}_{g}(M)$, i.e., a field of $g$-conformal frames which we use to identify any tangent space $T_{x} M$ with $W=\mathbb{R}^{n}$ and define the 1 -form

$$
\left.\omega^{-1}\right|_{x}(w)=\left(w^{1}, \ldots, w^{n}, 0, \ldots, 0\right), \quad w=\left(w^{i}\right) \in W \simeq T_{x} M, \quad x \in \mathcal{U} .
$$

By the observation before Definition 3.2, we may assume without loss of generality that $s$ is a field of $g$-orthonormal frames (in particular $g$ is identified at any point with the standard scalar product $\left.\left.\langle\cdot, \cdot\rangle\right|_{W}\right)$;

2. a co( $V)$-valued local 1 -form $\omega^{0}$ satisfying the equation

$$
\left[\omega^{-1}, \omega^{0}\right]=-d \omega^{-1}
$$

3. a $V^{*}$-valued local 1 -form $\omega^{1}$ satisfying the equations

$$
\begin{aligned}
& {\left[\omega^{-1}, \omega^{1}\right]=-\Omega^{0}=-d \omega^{0}-\frac{1}{2}\left[\omega^{0}, \omega^{0}\right],} \\
& \Omega^{1}=d \omega^{1}+\left[\omega^{1}, \omega^{0}\right]=0 .
\end{aligned}
$$

According to (5.12), we decompose $\omega^{0}=\omega^{\mathfrak{s o}(W)}+\omega^{W^{\perp} \otimes W^{*}}+\omega^{\mathfrak{s o}\left(W^{\perp}\right)}+\omega^{\mathbb{R}}$ and (5.14) is equivalent to the following equations for any $x \in \mathcal{U}, w_{1}, w_{2} \in W$ :

$$
\begin{aligned}
& -\left.d \omega^{-1}\right|_{x}\left(w_{1}, w_{2}\right)=-\omega_{x}^{\mathfrak{s o}(W)}\left(w_{2}\right)\left(w_{1}\right)+\omega_{x}^{\mathfrak{s o}(W)}\left(w_{1}\right)\left(w_{2}\right) \\
& -\omega_{x}^{\mathbb{R}}\left(w_{2}\right)\left(w_{1}\right)+\omega_{x}^{\mathbb{R}}\left(w_{1}\right)\left(w_{2}\right), \\
& 0=-\omega_{x}^{W^{\perp} \otimes W^{*}}\left(w_{2}\right)\left(w_{1}\right)+\omega_{x}^{W^{\perp} \otimes W^{*}}\left(w_{1}\right)\left(w_{2}\right) .
\end{aligned}
$$


From now on, we consider the solution of (5.17) determined by $\omega^{\mathbb{R}}=0$ and the connection 1form $\omega^{\mathfrak{s o}(W)}$ of $\nabla^{L C}$. Equation (5.18) means that $\omega^{W^{\perp} \otimes W^{*}}$ can be identified with a symmetric local section $\Pi$ of $\otimes^{2} T M^{*} \otimes N M$. Finally, we observe that $\omega^{\mathfrak{s o}\left(W^{\perp}\right)}$ is the connection 1-form of a metric connection $\nabla^{\perp}$ on $N M$.

Since $\omega^{\mathbb{R}}=0$, we can argue in complete analogy with Sect. 5.1 and decompose $\Omega^{0}$ according to (5.12)

$$
\Omega^{0}=\Omega^{\mathfrak{s o}(W)}+\Omega^{W^{\perp} \otimes W^{*}}+\Omega^{\mathfrak{s o}\left(W^{\perp}\right)} .
$$

The explicit expressions of each component $\Omega^{\mathfrak{s o}(W)}, \Omega^{W^{\perp} \otimes W^{*}}$, and $\Omega^{\mathfrak{s o}\left(W^{\perp}\right)}$ are as in Sect. 5.1 .

Further, according to (5.13), we decompose $\omega^{1}=\omega^{W^{*}}+\omega^{\left(W^{\perp}\right)^{*}}$ so that equation (5.15) splits into the following set of equations

$$
\begin{aligned}
\Omega^{\mathfrak{s o}(W)}\left(w_{1}, w_{2}\right)= & -w_{2} \otimes \omega^{W^{*}}\left(w_{1}\right)+\llbracket\left(\omega^{W^{*}}\left(w_{1}\right) \otimes w_{2}\right) \\
& +w_{1} \otimes \omega^{W^{*}}\left(w_{2}\right)-\sharp\left(\omega^{W^{*}}\left(w_{2}\right) \otimes w_{1}\right), \\
\Omega^{W^{\perp} \otimes W^{*}}\left(w_{1}, w_{2}\right)= & \natural\left(\omega^{\left(W^{\perp}\right)^{*}}\left(w_{1}\right) \otimes w_{2}\right)-\natural\left(\omega^{\left(W^{\perp}\right)^{*}}\left(w_{2}\right) \otimes w_{1}\right), \\
\Omega^{\mathfrak{s o}\left(W^{\perp}\right)}\left(w_{1}, w_{2}\right)= & 0, \\
\omega^{W^{*}}\left(w_{1}\right)\left(w_{2}\right)= & \omega^{W^{*}}\left(w_{2}\right)\left(w_{1}\right) .
\end{aligned}
$$

If we denote by $B$ (respectively, $D$ ) the local section of $\otimes^{2} T^{*} M$ (respectively, $T^{*} M \otimes N M$ ) which corresponds to the local 1 -form $\omega^{W^{*}}$ (respectively, $\omega^{\left(W^{\perp}\right)^{*}}$ ), one can check that the above equations correspond to (5.7), (5.8), (5.9) and the fact that $B$ is symmetric.

Now, according to (5.13), we decompose $\Omega^{1}$ into $\Omega^{1}=\Omega^{W^{*}}+\Omega^{\left(W^{\perp}\right)^{*}}$. A simple computation shows

$$
\begin{aligned}
\Omega^{W^{*}} & =d \omega^{W^{*}}-\omega^{W^{*}} \cdot \omega^{\mathfrak{s o}(W)}-\omega^{\left(W^{\perp}\right)^{*}} \cdot \omega^{W^{\perp} \otimes W^{*}}, \\
\Omega^{\left(W^{\perp}\right)^{*}} & =d \omega^{\left(W^{\perp}\right)^{*}}-\omega^{W^{*}} \cdot\left(\omega^{W^{\perp} \otimes W^{*}}\right)^{t}-\omega^{\left(W^{\perp}\right)^{*}} \cdot \omega^{\mathfrak{s o}\left(W^{\perp}\right)} .
\end{aligned}
$$

Equation (5.16) splits into $\Omega^{W^{*}}=0$ and $\Omega^{\left(W^{\perp}\right)^{*}}=0$. Using Eqs. (5.7)-(5.9), one can check that the first equation corresponds to (5.10) and the second one to (5.11). By these observations and the general results of Sect. 3.2, it follows that a Riemannian manifold $(M, g)$ with a triple $\left(\nabla^{\perp}, \Pi, B, D\right)$ as in (i)-(iv) and satisfying (5.7)-(5.11) has a local conformal immersion in $\left(S^{\widetilde{n}}, \widetilde{g}\right)$. This concludes the proof of the "if" direction of the Theorem.

The "only if" direction follows from Theorem 5.3 and the fact that $S^{\widetilde{n}}$ is locally conformal to $\mathbb{R}^{\tilde{n}}$.

The results in Sects. 3.3 and 4 allow to improve the above theorem and show that in many cases, the system (5.7)-(5.11) is equivalent to a smaller one.

Theorem 5.5 Under the assumption of Theorem 5.4, we have that:

(i) If $n \geq 4$, any solution $\left(\nabla^{\perp}, \Pi, B, D\right)$ of (5.7), (5.8), and (5.9) automatically satisfies (5.10) and (5.11) as well;

(ii) If $\tilde{n}=n+1$, local conformal immersions as hypersurfaces exist if and only if there are tensors $\Pi, B, D$ as in Theorem 5.4 satisfying (5.7), (5.8), (5.10), and (5.11) with $\nabla^{\perp}$ the flat connection. Moreover,

(a) if $n=2$, there is always a solution $(\Pi, B, D)$ of (5.7) and (5.8), 
(b) if $n \geq 4$, any solution ( $,, B, D)$ of (5.7), (5.8) automatically satisfies (5.10) and (5.11) as well.

Proof We first determine the cohomology groups $H^{p, 2}(\widetilde{\mathfrak{h}}, W)$ associated with $\widetilde{\mathfrak{h}}=$ $\mathfrak{s o}_{\tilde{n}+1,1}(\mathbb{R})$ and $W=\mathbb{R}^{n}$, for any $n \leq \widetilde{n}$. As $\widetilde{\mathfrak{h}}^{2}=0$, the only relevant cases are $p=0,1$ and 2 . We observe that if $n=\widetilde{n}$, these groups coincide with the usual Spencer groups $H^{p, 2}\left(\mathfrak{c o}_{n}(\mathbb{R})\right)$ of $\mathfrak{c o}_{n}(\mathbb{R})$ and $($ see $[15])$

$$
H^{p, 2}\left(\mathfrak{c o}_{n}(\mathbb{R})\right) \neq 0 \quad \text { only when } \quad \text { i) } p=2, n=3 \text { and } \quad \text { ii) } p=1, n \geq 4 .
$$

Further, for any $n<\tilde{n}$, one can directly check that $H^{0,2}(\widetilde{\mathfrak{h}}, W)=0$ (this is equivalent to the fact that (5.17) and (5.18) are always solvable).

Consider an element $\omega^{1} \in C^{2,2}(\widetilde{\mathfrak{h}}, W)=V^{*} \otimes \Lambda^{2} W^{*}$ and decompose it according to (5.13) into $\omega^{1}=\omega^{W^{*}}+\omega^{\left(W^{\perp}\right)^{*}}$. From definitions, it follows

$$
\partial \omega^{W^{*}} \in \mathfrak{c o}(W) \otimes \Lambda^{3} W^{*}, \quad \partial \omega^{\left(W^{\perp}\right)^{*}} \in W^{\perp} \otimes W^{*} \otimes \Lambda^{3} W^{*},
$$

so that the vanishing of $\partial \omega^{1}=\partial \omega^{W^{*}}+\partial \omega^{\left(W^{\perp}\right)^{*}}$ is equivalent to

$$
\partial \omega^{W^{*}}=0
$$

and

$$
\partial \omega^{\left(W^{\perp}\right)^{*}}=0 .
$$

If $n=2, \Lambda^{3} W^{*}=0$ so that (5.19) and (5.20) are always trivially satisfied. If $n \geq 3$, one can directly show that (5.20) implies $\omega^{\left(W^{\perp}\right)^{*}}=0$.

As $\widetilde{\mathfrak{h}}^{2}=0$, one has $H^{2,2}(\widetilde{\mathfrak{h}}, W)=Z^{2,2}(\widetilde{\mathfrak{h}}, W)$, and by the above discussion,

$$
H^{2,2}(\widetilde{\mathfrak{h}}, W) \simeq \begin{cases}H^{2,2}\left(\mathfrak{c o}_{n}(\mathbb{R})\right) & \text { if } n \geq 3 \\ V^{*} \otimes \Lambda^{2} W^{*} & \text { if } n=2 .\end{cases}
$$

In particular, $H^{2,2}(\widetilde{\mathfrak{h}}, W)$ is trivial when $n \geq 4$, for any $\widetilde{n}$.

Consider now an element $\omega^{0} \in C^{1,2}(\widetilde{\mathfrak{h}}, W)=\mathfrak{c o}(V) \otimes \Lambda^{2} W^{*}$ and decompose it according to $(5.12)$ into $\omega^{0}=\omega^{\mathfrak{s o}(W)}+\omega^{W^{\perp} \otimes W^{*}}+\omega^{\mathfrak{s o}\left(W^{\perp}\right)}+\omega^{\mathbb{R}}$. Equation $\partial \omega^{0}=0$ splits into the following two equations

$$
\partial \omega^{\mathfrak{s o}(W)}+\partial \omega^{\mathbb{R}}=0 \quad \text { and } \quad \partial \omega^{W^{\perp} \otimes W^{*}}=0 .
$$

Let also $\omega^{1} \in C^{2,1}(\widetilde{\mathfrak{h}}, W)=V^{*} \otimes W^{*}$ and decompose it according to (5.13) into $\omega^{1}=$ $\omega^{W^{*}}+\omega^{\left(W^{\perp}\right)^{*}}$. Using (5.6), one can check that the equation $\partial \omega^{1}=\omega^{0}$ is equivalent to

$$
\partial \omega^{W^{*}}=\omega^{\mathfrak{s o}(W)}+\omega^{\mathbb{R}} \quad \text { and } \quad \partial \omega^{\left(W^{\perp}\right)^{*}}=\omega^{W^{\perp} \otimes W^{*}} .
$$

The above discussion together with a direct computation and a dimensional argument shows that

$$
H^{1,2}(\widetilde{\mathfrak{h}}, W) \simeq\left\{\begin{array}{l}
H^{1,2}\left(\mathfrak{c o}_{n}(\mathbb{R})\right)+W^{\perp} \otimes \frac{\mathrm{R}^{2,1}}{W^{*}}+\mathfrak{s o}\left(W^{\perp}\right) \otimes \Lambda^{2} W^{*} \quad \text { if } n \geq 3, \\
W^{\perp} \otimes \frac{\mathrm{R}^{2,1}}{W^{*}}+\mathfrak{s o}\left(W^{\perp}\right) \otimes \Lambda^{2} W^{*} \text { if } n=2,
\end{array}\right.
$$

and

$$
\operatorname{dim} \frac{\mathrm{R}^{2,1}}{W^{*}}=\frac{n^{3}-4 n}{3}
$$


where $\mathrm{R}^{2,1} \subset W^{*} \otimes \Lambda^{2} W^{*}$ is the kernel of complete antysimmetrization. In particular, $H^{1,2}(\widetilde{\mathfrak{h}}, W)$ is trivial when $n=2, \widetilde{n}=3$.

Point (i) of the theorem is a direct consequence of (5.21), Theorem 3.4, the proof of Theorem 5.4, and finally, of the observation that the essential curvature $\left[\Omega^{1}\right]$ is naturally identified with the total curvature $\Omega^{1}$. Point (ii) of the theorem follows similarly from (5.21) and (5.22) and by applying Theorem 3.4 and Theorem 5.4 when $\widetilde{n}=n+1, W^{\perp}$ is 1 dimensional and $\mathfrak{s o}\left(W^{\perp}\right)=0$.

Remark 5.6 If $n=3$, neither the group $H^{1,2}(\widetilde{\mathfrak{h}}, W)$ nor $H^{2,2}(\widetilde{\mathfrak{h}}, W)$ vanishes, and the system (5.7)-(5.11) cannot be reduced simply as in Theorem 5.5.

\subsection{CR immersions into $\mathbb{C}^{n}$}

Let $(\widetilde{M}, \widetilde{J})$ be an almost complex manifold, a real manifold of even dimension $\widetilde{n}=2 \widetilde{m}$ with a tensor $\widetilde{J}: T \widetilde{M} \rightarrow T \widetilde{M}$ satisfying $\widetilde{J}^{2}=-I$. Fix an integer $0 \leq k \leq \widetilde{m}-1$ and consider the $\widetilde{G}$-structure on $\widetilde{M}, \widetilde{G} \simeq \mathrm{GL}_{\widetilde{m}}(\mathbb{C})$,

$$
\begin{gathered}
\widetilde{P}_{\widetilde{J}}=\left\{\left(e_{i}\right) \in L(\widetilde{M}) \mid e_{\widetilde{m}-k+i}=\widetilde{J} e_{i} \text { for any } 1 \leq i \leq \widetilde{m}-k\right. \text { and } \\
\left.e_{2 \widetilde{m}-k+i}=\widetilde{J} e_{2(\widetilde{m}-k)+i} \text { for any } 1 \leq i \leq k\right\} .
\end{gathered}
$$

One may check that a generic submanifold $M \subset \widetilde{M}$ of dimension $n=\widetilde{n}-k$ is $\widetilde{P}$-regular, and it is endowed with

(i) a rank $2(\widetilde{m}-k)$ distribution $\mathcal{D} \subset T M$,

(ii) a tensor $J: \mathcal{D} \rightarrow \mathcal{D}$ satisfying $J^{2}=-I$,

that is, $(M, \mathcal{D}, J)$ is an almost $\mathrm{CR}$ manifold of $\mathrm{CR}$ codimension $k$. The induced $G$-structure on $M$ is

$$
\begin{gathered}
P_{\mathcal{D}, J}=\left\{\left(e_{i}\right) \in L(M) \mid e_{i} \in \mathcal{D} \text { if } 1 \leq i \leq 2(\widetilde{m}-k)\right. \text { and } \\
\left.e_{\widetilde{m}-k+j}=J e_{j} \text { if } 1 \leq j \leq \widetilde{m}-k\right\} .
\end{gathered}
$$

In this case, the group $N_{\sharp}$ defined in (1.2) is trivial so that $G$ is naturally identified with the group $G_{\sharp}$ defined in (1.1).

It follows that, given an almost CR manifold $(M, \mathcal{D}, J)$ of real dimension $n=\widetilde{n}-k$ and CR codimension $k$ and an almost complex manifold $(\widetilde{M}, \widetilde{J})$ of real dimension $\widetilde{n}=2 \widetilde{m}$, the existence of a local CR immersion of $(M, \mathcal{D}, J)$ into $(\widetilde{M}, \widetilde{J})$ is equivalent to the existence of a local immersion of the associated bundle $P_{\mathcal{D}, J}$ into the bundle $\widetilde{P}_{\widetilde{J}}$ of $\widetilde{M}$. In particular, the existence of a local CR immersion of $(M, \mathcal{D}, J)$ into $\mathbb{C}^{\widetilde{m}}$, endowed with the standard complex structure $\widetilde{J}_{o}$, is equivalent to the local immersibility of $\pi: P_{\mathcal{D}, J} \rightarrow M$ into the $\widetilde{G}$-structure $\widetilde{\pi}: \widetilde{P}_{\widetilde{J}_{o}} \rightarrow \mathbb{C}^{\widetilde{m}}$.

The latter is homogeneous and flat but it is not of finite type, since the maximal prolongation $\widetilde{\mathfrak{h}}_{\infty}=\sum_{p=-1}^{\infty} \widetilde{\mathfrak{h}}_{\infty}^{p}$ of $\widetilde{\mathfrak{g}}=\mathfrak{g l}_{\widetilde{m}}(\mathbb{C})$ is infinite dimensional (see, e.g., [9]). However, the notions of immersibility to order $p$ and of generalized cohomology still make sense, and in the real analytic setting, immersibility at any order still yields complete immersibility $([7,16])$.

The next proposition provides the relevant cohomology groups. We first need to fix some notation. Let $W \simeq \mathbb{R}^{n}$ (respectively, $U \simeq \mathbb{R}^{2(\widetilde{m}-k)}$ ) be the subspace of $V=\mathbb{R}^{\widetilde{n}}$ determined by the vanishing of the last $k$ (respectively, $2 k$ ) standard coordinates. We also denote by $W^{\perp}$ (respectively, $U^{\perp}$ ) the subspace of $V$ (respectively, $W$ ) determined by the vanishing of the first $n$ (respectively, $2(\tilde{m}-k)$ ) standard coordinates of $V$. There are vector space direct sum decompositions

$$
V=W+W^{\perp} \simeq \mathbb{R}^{n}+\mathbb{R}^{k}, \quad W=U+U^{\perp} \simeq \mathbb{R}^{2(\widetilde{m}-k)}+\mathbb{R}^{k} .
$$


Proposition 5.7 All groups $H^{p, 2}\left(\widetilde{\mathfrak{h}}_{\infty}, W\right), p \geq 1$ are trivial.

Proof We recall that the $(p-1)$ th-component of the maximal prolongation $\widetilde{\mathfrak{h}}_{\infty}$ of $\widetilde{\mathfrak{g}}=\mathfrak{g l}_{\widetilde{m}}(\mathbb{C})$ is $\widetilde{\mathfrak{h}}_{\infty}^{p-1}=\mathbb{C}^{\widetilde{m}} \otimes_{\mathbb{C}} S^{p}\left(\mathbb{C}^{\widetilde{m}}\right)^{*}$, and it has a natural structure of complex vector space (see, e.g., [9]).

We also note that, since any $v \in W^{\perp}$ satisfies $\widetilde{J}_{o} v \in U^{\perp} \subset W$, it is possible to extend any element $\omega^{p-1} \in C^{p, 2}\left(\widetilde{\mathfrak{h}}_{\infty}, W\right)=\widetilde{\mathfrak{h}}_{\infty}^{p-1} \otimes \Lambda^{2} W^{*}$ to an element $\widetilde{\omega}^{p-1} \in C^{p, 2}\left(\widetilde{\mathfrak{h}}_{\infty}, V\right)=$ $\widetilde{\mathfrak{h}}_{\infty}^{p-1} \otimes \Lambda^{2} V^{*}$, setting

$$
\begin{array}{ll}
\widetilde{\omega}^{p-1}\left(v_{1}, v_{2}\right):=-\omega^{p-1}\left(\widetilde{J}_{o} v_{1}, \widetilde{J}_{o} v_{2}\right) & \text { for all } v_{1}, v_{2} \in W^{\perp}, \\
\widetilde{\omega}^{p-1}\left(v_{1}, w_{2}\right):=-i \omega^{p-1}\left(\widetilde{J}_{o} v_{1}, w_{2}\right) & \text { for all } v_{1} \in W^{\perp}, w_{2} \in W .
\end{array}
$$

We finally observe that the generalized Spencer operator on $C^{p, 2}\left(\widetilde{\mathfrak{h}}_{\infty}, V\right)$ is just the usual Spencer operator and that the corresponding cohomology $H^{p, 2}\left(\widetilde{\mathfrak{h}}_{\infty}, V\right)$ is just the Spencer group $H^{p, 2}\left(\mathfrak{g l}_{\widetilde{m}}(\mathbb{C})\right)$ of $\mathfrak{g l}_{\widetilde{m}}(\mathbb{C})$. It is known that this group is trivial for any $p \geq 1([7,12])$.

Now, one can check that if $\partial \omega^{p-1}=0$, then also $\partial \widetilde{\omega}^{p-1}=0$. From this fact, for all $p \geq 1$ and $\omega^{p-1}$ with $\partial \omega^{p-1}=0$, one has

$$
\begin{array}{lll}
\widetilde{\omega}^{p-1}=\partial \widetilde{\omega}^{p} & \text { for some } & \widetilde{\omega}^{p} \in C^{p+1,1}\left(\widetilde{\mathfrak{h}}_{\infty}, V\right) \quad \text { so that } \\
\omega^{p-1}=\partial \omega^{p} & \text { where } & \omega^{p}=\left.\widetilde{\omega}^{p}\right|_{W} \in C^{p+1,1}\left(\widetilde{\mathfrak{h}}_{\infty}, W\right) .
\end{array}
$$

In other words, $H^{p, 2}\left(\widetilde{\mathfrak{h}}_{\infty}, W\right)=0$ for every $p \geq 1$.

Now, we observe that any coboundary $T \in B^{0,2}\left(\widetilde{\mathfrak{h}}_{\infty}, W\right)$ is the restriction to $\Lambda^{2} W$ of some $\widetilde{T} \in B^{0,2}\left(\widetilde{\mathfrak{h}}_{\infty}, V\right)$ and

$$
\begin{aligned}
B^{0,2}\left(\widetilde{\mathfrak{h}}_{\infty}, V\right)= & \left\{\widetilde{T} \in V \otimes \Lambda^{2} V^{*} \mid \text { for every } v_{1}, v_{2} \in V\right. \\
& \left.\widetilde{T}\left(v_{1}, v_{2}\right)-\widetilde{T}\left(\widetilde{J}_{o} v_{1}, \widetilde{J}_{o} v_{2}\right)=-\widetilde{J}_{o} \widetilde{T}\left(\widetilde{J}_{o} v_{1}, v_{2}\right)-\widetilde{J_{o}} \widetilde{T}\left(v_{1}, \widetilde{J}_{o} v_{2}\right)\right\} .
\end{aligned}
$$

From this, one gets that the vector space

$$
B^{0,2}\left(\widetilde{\mathfrak{h}}_{\infty}, W\right) \cap W \otimes \Lambda^{2} W^{*}
$$

is formed by all maps $T \in W \otimes \Lambda^{2} W^{*}$ satisfying

$$
\begin{aligned}
& T\left(u_{1}, u_{2}\right)-T\left(\widetilde{J}_{o} u_{1}, \widetilde{J}_{o} u_{2}\right) \in U, \\
& T\left(u_{1}, u_{2}\right)-T\left(\widetilde{J}_{o} u_{1}, \widetilde{J}_{o} u_{2}\right)=-\widetilde{J}_{o} T\left(\widetilde{J}_{o} u_{1}, u_{2}\right)-\widetilde{J}_{o} T\left(u_{1}, \widetilde{J}_{o} u_{2}\right),
\end{aligned}
$$

for any $u_{1}, u_{2} \in U$. By Proposition 5.7, all the essential curvatures of order bigger than 1 of an admissible tuple are trivial. Thus, an analytic almost CR manifold has a local CR immersion in $\mathbb{C}^{\widetilde{m}}$ if and only if the total curvature

$$
\Omega^{-1}: \mathcal{U} \subset M \rightarrow Z^{0,2}\left(\widetilde{\mathfrak{h}}_{\infty}, W\right)
$$

takes values in the space of coboundaries $B^{0,2}\left(\widetilde{\mathfrak{h}}_{\infty}, W\right)$. We remark that, by its very definition, the map (5.24) always takes values in $W \otimes \Lambda^{2} W^{*}$. It follows that $(M, \mathcal{D}, J)$ is locally immersible in $\mathbb{C}^{\widetilde{m}}$ if and only if (5.24) takes values in $B^{0,2}\left(\widetilde{\mathfrak{h}}_{\infty}, W\right) \cap W \otimes \Lambda^{2} W^{*}$ and, by Eq. (5.23), if and only if

$$
\begin{aligned}
& {[X, Y]-[J X, J Y] \in \mathcal{D} \quad \text { and }} \\
& {[X, Y]-[J X, J Y]=-J[J X, Y]-J[X, J Y],}
\end{aligned}
$$

for all sections $X, Y$ of $\mathcal{D}$, i.e., when the almost CR structure is integrable in the classical sense. We therefore reobtained the classical result by Andreotti and Hill ([5]) on immersibility of almost CR structures. 


\subsection{CR quaternionic immersions into $\mathbb{H}^{n}$}

Let $(\widetilde{M}, Q)$ be an almost quaternionic manifold, a real manifold of dimension $4 \widetilde{n}$ with a subbundle $Q$ of End $(T \widetilde{M})$ locally generated by a triple $\left(J_{1}, J_{2}, J_{3}\right)$ of endomorphisms satisfying

$$
J_{3}=J_{1} J_{2}, \quad J_{\alpha}^{2}=-I, \quad J_{\alpha} \circ J_{\beta}=-J_{\beta} \circ J_{\alpha} \quad \text { for all } 1 \leq \alpha, \beta \leq 3, \quad \alpha \neq \beta .
$$

To any $(\widetilde{M}, Q)$, one can naturally associate a $\widetilde{G}$-structure $\widetilde{\pi}: \widetilde{P} \rightarrow \widetilde{M}$ with $\widetilde{G}=\mathrm{GL}_{\widetilde{n}}(\mathbb{H}) \cdot \mathrm{Sp}_{1}$ (see $[3,17])$.

Let now $M$ be a real manifold of dimension $4 \widetilde{n}-1$ endowed with an embedding $T M \hookrightarrow E$ of its tangent bundle into a vector bundle $E \rightarrow M$ of $\operatorname{rank}(E)=4 \tilde{n}$ with a linear quaternionic structure on each fiber. Following [13], the pair $(M, E)$ is an almost CR quaternionic manifold (of hypersurface type). To any such manifold, one can naturally associate a $G$-structure $\pi: P \rightarrow M$ with a particular structure group $G$ which is not semisimple and has Levi factor isomorphic with $\mathrm{SL}_{\widetilde{n}-1}(\mathbb{H}) \times \mathrm{Sp}_{1}$. We refer to [18] for its explicit description.

Any hypersurface $M$ of an almost quaternionic manifold $\widetilde{M}$ is $\widetilde{P}$-regular and inherits an almost CR quaternionic structure, the natural inclusion $T M \subset E:=\left.T \widetilde{M}\right|_{M}$. Moreover, the above-mentioned bundle $\pi: P \rightarrow M$ coincides with the $G$-structure induced on $M \subset \widetilde{M}$ by $\tilde{\pi}: \widetilde{P} \rightarrow \widetilde{M}$.

It follows that, given an almost CR quaternionic manifold $(M, E)$ of dimension $4 \tilde{n}-1$ and an almost quaternionic manifold $(\widetilde{M}, Q)$ of dimension $4 \widetilde{n}$, the existence of a local CR quaternionic immersion of $(M, E)$ into $(\widetilde{M}, Q)$ is equivalent to the local immersibility of $\pi: P \rightarrow M$ into $\widetilde{\pi}: \widetilde{P} \rightarrow \widetilde{M}$. In particular, the existence of a local CR quaternionic immersion of $(M, E)$ in the quaternionic projective space $\widetilde{M}=\mathbb{H} \mathrm{P}^{\widetilde{n}}$ with its standard quaternionic structure $Q=Q_{o}$ is equivalent to the local immersibility of $\pi: P \rightarrow M$ in the $\widetilde{G}$-structure $\widetilde{\pi}: \widetilde{P}_{o} \rightarrow \mathbb{H} \mathrm{P}^{\tilde{n}}$ associated with $\left(\mathbb{H} \mathrm{P}^{\tilde{n}}, Q_{o}\right)$. This structure is flat $k$ reductive with $k=2$. We refer to [18] for the explicit expression of the maximal prolongation $\widetilde{\mathfrak{h}}_{\infty}$ of $\widetilde{\mathfrak{g}}=\mathfrak{g l}_{\tilde{n}}(\mathbb{H})+\mathfrak{s p}_{1}$ and its main properties. In that paper, it is also proved that the groups $H^{1,1}\left(\widetilde{\mathfrak{h}}_{\infty}, W\right)$ and $H^{2,1}\left(\widetilde{\mathfrak{h}}_{\infty}, W\right)$ are trival. This fact, a careful analysis of the groups $H^{p, 2}\left(\widetilde{\mathfrak{h}}_{\infty}, W\right)$ and Theorems 1.1, 3.4 and 4.3, yield the following result on CR quaternionic immersions. In the statement,

- $\mathbf{E}=\mathbb{C}^{2 \widetilde{n}-2}$ and $\mathbf{H}=\mathbb{C}^{2}$ are the standard representations of $\mathfrak{s l}_{2 \widetilde{n}-2}(\mathbb{C})$ and $\mathfrak{s l}_{2}(\mathbb{C})$, respectively,

- Ad is the adjoint representation of $\mathfrak{s l}_{2 \widetilde{n}-2}(\mathbb{C})$,

- D is the $\mathfrak{s l}_{2 \widetilde{n}-2}(\mathbb{C})$-irreducible module given by the kernel of the natural contraction $\mathbf{E} \otimes \Lambda^{2} \mathbf{E}^{*} \rightarrow \mathbf{E}^{*}$.

Theorem 5.8 Let $M$ be an almost $C R$ quaternionic manifold of dimension $4 \tilde{n}-1 \geq 7$ and $\pi: P \rightarrow M$ its canonically associated $G$-structure. Then:

(i) there are natural isomorphisms of $\mathfrak{s}$-modules, $\mathfrak{s} \simeq \mathfrak{s l}_{2 \widetilde{n}-2}(\mathbb{C})+\mathfrak{s l}_{2}(\mathbb{C})$,

$$
\begin{aligned}
& H^{0,2}\left(\widetilde{\mathfrak{h}}_{\infty}, W\right) \otimes \mathbb{C} \simeq \Lambda^{2} \boldsymbol{E}^{*} S^{2} \boldsymbol{H}+\left(\boldsymbol{D}+\boldsymbol{E}^{*}\right) S^{3} \boldsymbol{H}+\left(\mathrm{Ad}+\Lambda^{2} \boldsymbol{E}^{*}\right) S^{4} \boldsymbol{H}+\boldsymbol{E}^{*} S^{5} \boldsymbol{H} \\
& H^{1,2}\left(\widetilde{\mathfrak{h}}_{\infty}, W\right) \otimes \mathbb{C} \simeq H^{1,2}(\mathfrak{g}) \otimes \mathbb{C},
\end{aligned}
$$

while the cohomology group $H^{2,2}\left(\widetilde{\mathfrak{h}}_{\infty}, W\right)$ vanishes;

(ii) there is a canonical $G$-equivariant map $\mathcal{R}^{1}: P \rightarrow H^{0,2}\left(\widetilde{\mathfrak{h}}_{\infty}, W\right)$ which vanishes if $M$ is locally immersible into a quaternionic manifold (i.e., an almost quaternionic manifold with a compatible torsion-free connection); 
(iii) if $\mathcal{R}^{1}=0$, there is a canonical $G$-equivariant map $\mathcal{R}^{2}: P \rightarrow H^{1,2}\left(\widetilde{\mathfrak{h}}_{\infty}, W\right)$;

(iv) $M$ is locally immersible in $\mathbb{H} \mathrm{P}^{n}$ around any point if and only if $\mathcal{R}^{1}=\mathcal{R}^{2}=0$.

Remark 5.9 The fact that the essential curvatures $\mathcal{R}^{1}$ and $\mathcal{R}^{2}$ of the theorem are intrinsically defined objects relies on the non-trivial fact that $H^{1,1}\left(\widetilde{\mathfrak{h}}_{\infty}, W\right)$ and $H^{2,1}\left(\widetilde{\mathfrak{h}}_{\infty}, W\right)$ vanish. In general, these groups do not vanish: consider the case of isometric immersions into Euclidean spaces where

$$
H^{1,1}\left(\mathfrak{s o}_{\tilde{n}}(\mathbb{R}), W\right) \simeq \underbrace{H^{1,1}\left(\mathfrak{s o}_{n}(\mathbb{R}), W\right)}_{=\mathfrak{s o}_{n}(\mathbb{R})^{1}=0}+W^{\perp} \otimes S^{2} W^{*}+\mathfrak{s o}\left(W^{\perp}\right) \otimes W^{*} .
$$

The nonvanishing of the last two subspaces corresponds to the fact that the existence of a local Riemannian immersion depends on the existence of an appropriate fundamental form and normal connection.

Acknowledgments This research was partially supported by the Project Firb 2012 Geometria differenziale e teoria geometrica delle funzioni, by GNSAGA of INdAM and by project F1R-MTH-PUL-08HALOHALOS08 of University of Luxembourg. Part of this work was done while the author was a post doc at the University of Parma. The author would like to thank the Mathematics Department and in particular A. Tomassini and C. Medori for support and ideal working conditions. The author is also grateful to A. Spiro for useful discussions on various aspects of this paper and to the anonymous referee for his helpful comments and suggestions.

\section{References}

1. Akivis, M.A.: On the conformal differential geometry of multidimensional surfaces. Mat. Sb. (N.S.) 53(95), 53-72 (1958). (Russian)

2. Akivis, M.A., Goldberg, V.V.: Conformal Differential Geometry and its Generalizations. WileyInterscience, New York (1999)

3. Alekseevsky, D.V., Marchiafava, S.: Quaternionic structures on a manifold and subordinated structures. Ann. Mat. Pura Appl. (4) 171, 205-273 (1996)

4. Alekseevsky, D.V., Spiro, A.F.: Generalized reductive homogeneous spaces and quasi-graded semisimple Lie algebras. In: Algebra and Analysis (Kazan, 1994), pp. 1-22, de Gruyter, Berlin (1996)

5. Andreotti, A., Hill, C.D.: Complex characteristic coordinates and tangential Cauchy-Riemann equations. Ann. Sc. Norm. Sup. Pisa 26, 299-324 (1979)

6. Besse, A.L.: Einstein Manifolds. Springer, Berlin (2008)

7. Guillemin, V.: The integrability problem for $G$-structures. Trans. Am. Math. Soc. 116, 544-560 (1965)

8. Kantor, I.L.: Transitive differential groups and invariant connections in homogeneous spaces. Trudy Sem. Vektor. Tenzor. Anal. 13, 310-398 (1966). (Russian)

9. Kobayashi, S.: Transformation Groups in Differential Geometry. Springer, New York (1972)

10. Kobayashi, S., Nagano, T.: On filtered Lie algebras and geometric structures I. J. Math. Mech. 13, 875-907 (1964)

11. Kobayashi, S., Nomizu, K.: Foundations of Differential Geometry. Interscience, New York (1963)

12. Kodaira, K., Spencer, D.C.: Multifoliate structures. Ann. Math. 74, 52-100 (1961)

13. Marchiafava, S., Ornea, L., Pantilie, R.: Twistor theory for CR quaternionic manifolds and related structures. Monatsh. Math. 167, 531-545 (2012)

14. Ochiai, T.: Classification of the finite nonlinear primitive Lie algebras. Trans. Am. Math. Soc. 124, 313322 (1966)

15. Ochiai, T.: Geometry associated with semisimple flat homogeneous spaces. Trans. Am. Math. Soc. 152, 159-193 (1970)

16. Rosly, A.A., Schwarz, A.S.: Geometry of N = 1 supergravity. II. Comm. Math. Phys. 96, 285-309 (1984)

17. Salamon, S.M.: Differential geometry of quaternionic manifolds. Ann. Sci. Ecole Norm. Sup. 19, 31-55 (1986)

18. Santi, A.: Almost CR quaternionic manifolds and their immersibility in $\mathbb{H}^{n}$, preprint arXiv:1311.4072 (2015)

19. Santi, A.: A generalized integrability problem for higher order G-structures (in preparation) 
20. Singer, I.M., Sternberg, S.: The infinite groups of Lie and Cartan I. The transitive groups. J. Anal. Math. 15, 1-114 (1965)

21. Spivak, M.: A Comprehensive Introduction to Differential Geometry, vol.4, third edition, Publish or Perish, (1999)

22. Sternberg, S.: Lectures on Differential Geometry. Prentice-Hall, Englewood Cliffs (1964) 